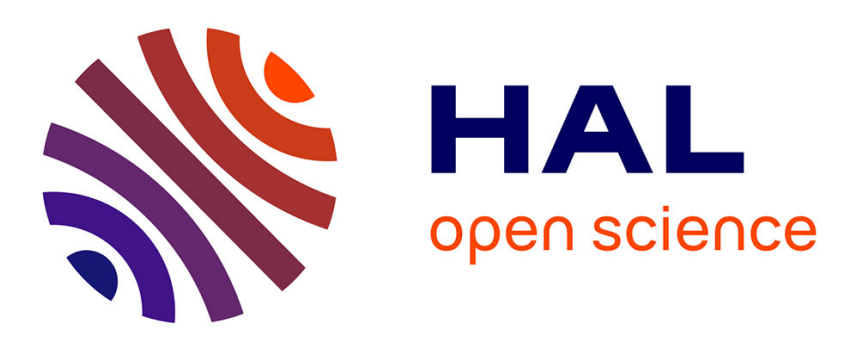

\title{
Sensitivity and error analysis of the dual criteria traffic assignment model
}

\author{
Fabien Leurent
}

\section{To cite this version:}

Fabien Leurent. Sensitivity and error analysis of the dual criteria traffic assignment model. Transportation Research Part B: Methodological, 1998, Vol. 32, pp. 189-204. hal-00348420

\section{HAL Id: hal-00348420 \\ https://hal.science/hal-00348420}

Submitted on 18 Dec 2008

HAL is a multi-disciplinary open access archive for the deposit and dissemination of scientific research documents, whether they are published or not. The documents may come from teaching and research institutions in France or abroad, or from public or private research centers.
L'archive ouverte pluridisciplinaire HAL, est destinée au dépôt et à la diffusion de documents scientifiques de niveau recherche, publiés ou non, émanant des établissements d'enseignement et de recherche français ou étrangers, des laboratoires publics ou privés. 


\title{
SENSITIVITY AND ERROR ANALYSIS OF THE DUAL CRITERIA TRAFFIC ASSIGNMENT MODEL
}

\author{
FABIEN M. LEURENT
}

INRETS, 2 avenue Malleret-Joinville, 94114 Arcueil Cedex, France

\begin{abstract}
In the dual criteria traffic assignment model, to take into account various trade-offs between travel time and travel cost, the value of time is assumed to be continuously distributed across the trip-makers. This paper presents a sensitivity and error analysis method for the equilibrium solution of a dual criteria model with elastic demand, flowdependent travel times and side constraints. The method is based on previous work by Tobin and Friesz (1988) to bypass the uniqueness requirement in standard sensitivity analysis frameworks for variational inequalities, and exploits the finite-dimensional formulation of the model in terms of path flow variables given by Leurent (1996a). The paper contains three parts. The first demonstrates the usefulness of sensitivity analysis using a two-link network. The second addresses the general network case. Lastly, the third presents an application to a realistic traffic study.
\end{abstract}

\section{KEYWORDS}

Assignment; Multicriteria Assignment; Sensitivity Analysis; Variational Inequality

\section{INTRODUCTION}

Context. A dual criteria traffic assignment model simulates the way trip-makers select a route from among the competing paths which are differentiated on the basis of two cost criteria, eg. time and financial cost. It is assumed that each trip-maker will minimize the generalized cost of his trip, the generalized cost being the sum of the cost and the time weighted by the value of time (VoT). In order to represent the different trade-offs made by trip-makers between price and cost it is assumed that the value of time is continuously distributed (Quandt, 1968; Marche, 1973; Leurent, 1993a among others).

As in the case of any quantitative simulation model, the following question naturally arises: what degree of confidence can one have in the model, in other terms how close is it to reality? Put yet another way, what is the modelling error? When a dual criteria 
assignment model is used to forecast traffic on a privately funded toll road, this question has particular economic relevance as an error of a few percent on traffic, and therefore on revenue, may place the financial equilibrium of the concession at risk.

Objective. Four types of modelling error can be identified (Leurent, 1996b). These are related to: (i) the design (which discretizations and which behavioural features are considered in the theoretical representation?), (ii) the mathematical formulation (representing the behavioural features as a standard mathematical programming problem), (iii) the computation (implementation and convergence if an algorithm is involved), (iv) econometric aspects (the exogenous error which affects the input data as well as the parameters).

The objective of this paper is to examine econometric error in a dual criteria assignment model: this model outputs an average value which is obtained on the basis of the average values of the inputs. In fact, the exogenous uncertainty which applies to these inputs (including the parameters) is propagated through the model and leads to uncertainty as regards the output. Our objective involves characterizing uncertainty in the output on the basis of uncertainty in the inputs, by expressing the output as a random variable whose probability distribution depends on the probability distribution of inputs. The paper provides formulae for propagating exogenous uncertainty for any input through a dual criteria assignment model with continuously distributed value of time (VoT), elastic demand, with variable travel times and side constraints, such as that formulated by Leurent (1996a). In particular we shall consider three types of a priori exogoneous error. These are the distribution of VoT, origin-destination (O-D) volumes and journey times.

This study of modelling error in the dual criteria model is limited in a number of respects. Firstly, we shall not deal with design error - in particular we consider neither queueing effects nor dynamic modifications in journey starting times or mode. We shall pay most attention to the various trade-offs between the two criteria in the generalized cost, thus on the continuous distribution of the VoT. We shall not deal with formulation or computation error here either: we shall assume that the mathematical formulation used in the model is correct and that it uses an algorithm which gives an accurate result. Finally, as regards the quantification of the a priori exogenous error, we shall simply take the numerical values which have been established elsewhere (Leurent 1996b).

Method. In order to analyze the sensitivity of a solution to finite-dimensional variational inequalities we shall make use of theorems which were developed by Tobin (1986) and then extended to the problems of equilibrium on a network by Tobin and Friesz (1988). A finite-dimensional variational formulation does in fact exist for the dual criteria assignment model which is considered here (Leurent 1993a, 1996a). In 
order to display the effects of the behavioural assumptions which are built into this model we shall begin with the simple case of a two arc network and progressively introduce the continuous distribution of VoT, the dependency of travel times on the level of traffic and finally the dependency of volume of demand on level of service.

It should be noted that two ingredients are necessary in order to carry out the analysis of sensitivity and error presented here: a finite-dimensional mathematical formulation, and the path results of assignment. Thus, infinite dimension formulations of the dual criteria model, e.g. Dafermos (1981), Leurent (1993b), Marcotte and Zhu (1994) and Dial (1996) cannot be used. The Frank-Wolfe algorithm which is advocated in the last two references does not identify paths and is unable to output assignment results in the necessary form, except by recalculating on an a posteriori basis the paths between which origin-destination (O-D) volumes are distributed at equilibrium.

Structure. The main body of the paper is in three sections. The first deals with the elementary case of a two arc network and shows how the gradual incorporation of explanatory mechanisms makes it possible to reduce econometric error in the model output. The second section deals with the general case of a network of unlimited size. Finally the third section describes an operational application to an interurban traffic study and shows the way in which uncertainty which relates to the traffic on one link depends on the uncertainty which relates to the journey times on all links, to the demand volumes on all O-D pairs and the distribution of VoT.

\section{THE CASE OF THE TWO ARC NETWORK}

Let us consider a network with two arcs which are competing routes linking a single origin to a single destination, which is the simplest possible case of competing routes. We shall assume that one of these routes, which we shall refer to as $F$, is free, while a toll $\mathrm{P}$ must be paid on the other, which we shall refer to as $\mathrm{T}$ (both routes could actually be toll routes, in which case $\mathrm{P}$ would be the difference in price).

The toll route is only competitive if its journey time, $T_{\mathrm{T}}$, is lower than that of the free route, $T_{\mathrm{F}}$. In this situation users with a low value of time select the free route on the grounds that it is inexpensive even though it is slow, whereas those users with a high value of time choose the toll route because it is fast even though it is expensive.

More formally, we shall assume that each trip-maker measures the (dis-)utility of a path $k$ with respect to his own VoT $v$ on the basis of the generalized travel cost expressed as $G_{k}(v)=\mathrm{P}_{k}+v \cdot T_{k}$, and further that he chooses a path with minimum disutility (optimizing behaviour assumption). Trips-makers with $v$ such that $G_{\mathrm{F}}(v)=v \cdot T_{\mathrm{F}}$ is less than, or equal to, $G_{\mathrm{T}}(v)=\mathrm{P}+v \cdot T_{\mathrm{T}}$ choose the free route, whereas those with $v$ such that 
$G_{\mathrm{T}}(v)$ is less than $G_{\mathrm{F}}(v)$ choose the toll route.

The cut-off VoT which separates the two user groups, $\hat{v}$, is that for which the generalized costs of the two routes are equal: $\widehat{v} \cdot T_{\mathrm{F}}=\mathrm{P}+\widehat{v} \cdot T_{\mathrm{T}}$, hence

$$
\widehat{v}=\mathrm{P} /\left(T_{\mathrm{F}}-T_{\mathrm{T}}\right) .
$$

If we let $\mathrm{H}$ denote the cumulative distribution function of $\mathrm{VoT}$, the proportion of users using the free route is $\operatorname{Pr}(\{v \leq \widehat{v}\})=\mathrm{H}(\widehat{v})$ whereas the market share of the toll route is $\operatorname{Pr}(\{v>\widehat{v}\})=1-\mathrm{H}(\widehat{v})$. For an O-D volume of $q$, the flow on the free route is $f_{\mathrm{F}}=q \mathrm{H}(\widehat{v})$ while the flow on the toll route is:

$$
f_{\mathrm{T}}=q(1-\mathrm{H}(\widehat{v})) .
$$

\subsection{Model with fixed travel times and fixed demand}

In this section we shall consider the flow on the toll route to be the output of the model. If we fix the journey times $T_{\mathrm{F}}$ and $T_{\mathrm{T}}$ as well as the demand volume $q$, the output depends on the exogenous variables $T_{\mathrm{F}}, T_{\mathrm{T}}, \mathrm{P}, q$ and $\mathrm{H}$ (which is a function). A variation $\delta \mathrm{X}$ which affects the input $\mathrm{X}$ results in a variation $\delta \mathrm{Y}$ in the output $\mathrm{Y}$. In the case of a model $\mathrm{Y}=\mathrm{F}(\mathrm{X})$, this gives (Tukey, 1957):

$$
\delta \mathrm{Y}=\nabla_{\mathrm{X}} \mathrm{F} . \delta \mathrm{X}
$$

which is the basic formula for sensitivity analysis and the propagation of (small) biases. In particular a variation $\delta \mathrm{X}$ may represent a sample of the exogenous error $\varepsilon_{\mathrm{X}}$, in which case $\delta \mathrm{Y}$ is a sample of the propagated exogenous error, $\varepsilon_{\mathrm{Y}}$. By squaring both sides of (3) and taking expectation over the error distributions, we obtain the basic formula for the propagation of (co-)variance (assuming there is no exogenous bias, i.e. $\left.\mathrm{E}\left(\varepsilon_{X}\right)=0\right)$ :

$$
\mathrm{E}\left(\varepsilon_{\mathrm{Y}}{ }^{2}\right)=\sum_{n, m}\left(\frac{\partial \mathrm{F}}{\partial X_{n}} \frac{\partial \mathrm{F}}{\partial X_{m}}\right) \operatorname{Cov}\left(\varepsilon_{X_{n}} ; \varepsilon_{X_{m}}\right),
$$

in which $\varepsilon_{X_{n}}$ is the exogenous error on the component $X_{n}$ of the vector X.

Sensitivity analysis of the two arc network. Formula (3) gives, for each component $X_{n}$ :

$$
\delta f_{\mathrm{T}}=\frac{\partial}{\partial X_{n}}\left(q\left(1-\mathrm{H}_{\Theta}\left(\frac{\mathrm{P}}{T_{\mathrm{F}}-T_{\mathrm{T}}}\right)\right)\right) \cdot \delta X_{n}
$$

where $\Theta$ is the vector of the parameters $\theta_{i}$ of the function $\mathrm{H}$. We shall consider the input data vector $\mathrm{X}={ }^{t}\left[T_{\mathrm{F}} ; T_{\mathrm{T}} ; q ; \theta_{i}\right]$. The following partial derivatives are therefore computed: 


$$
\begin{aligned}
& \frac{\partial f_{\mathrm{T}}}{\partial T_{\mathrm{F}}}=-\frac{\partial f_{\mathrm{T}}}{\partial T_{\mathrm{T}}}=-q \frac{\partial \mathrm{H}}{\partial v}(\hat{v}) \frac{\partial \hat{v}}{\partial T_{\mathrm{F}}}=q \frac{\mathrm{P}}{\left(T_{\mathrm{F}}-T_{\mathrm{T}}\right)^{2}} \frac{\partial \mathrm{H}}{\partial v}(\hat{v}), \\
& \partial f_{\mathrm{T}} / \partial q=1-\mathrm{H}(\hat{v})=f_{\mathrm{T}} / q, \\
& \frac{\partial f_{\mathrm{T}}}{\partial \theta_{i}}=-q \frac{\partial \mathrm{H}}{\partial \theta_{i}}(\hat{v}) .
\end{aligned}
$$

The partial, rather than total, derivative of $\mathrm{H}$ with respect to the VoT $v$ is considered because $\mathrm{H}_{\Theta}(v)$ also depends on the parameters $\theta_{i}$.

Numerical illustration. Let $\mathrm{P}=3 \$, q=3000 \mathrm{veh} / \mathrm{h}, T_{\mathrm{F}}=0.430 \mathrm{~h}, T_{\mathrm{T}}=0.216 \mathrm{~h}$, and take a value of time whose natural logarithm has a normal distribution with mean $\mu=$ 2.48 and standard deviation $\sigma=0.6$. Thus the average VoT is $M=12 \$ / \mathrm{h}$ as it is given by $M=\exp \left(\mu+\sigma^{2} / 2\right)$. The cut-off VoT is $\hat{v}=14.0 \$ / \mathrm{h}$ therefore $\mathrm{H}(\hat{v})=0.71$ and the market share of the toll route is $f_{\mathrm{T}} / q=0.29$. The values of the partial derivatives of $\mathrm{H}$ are as follows: $\partial \mathrm{H} / \partial v=0.0082, \partial \mathrm{H} / \partial \mu=-0.57$ and $\partial \mathrm{H} / \partial \sigma=-0.32$ (see Appendix). Thus the output partial derivatives and elasticities are:

$$
\begin{array}{lll}
\partial f_{\mathrm{T}} / \partial T_{\mathrm{F}}=-\partial f_{\mathrm{T}} / \partial T_{\mathrm{T}}=7968 \mathrm{veh} / \mathrm{h}^{2} & \text { and } & e_{\Delta T}=\partial \ln f_{\mathrm{T}} / \partial \ln \left(T_{\mathrm{F}}-T_{\mathrm{T}}\right)=1.97 \\
\partial f_{\mathrm{T}} / \partial q=0.29 & \text { and } & e_{q}=1 \\
\partial f_{\mathrm{T}} / \partial \mu=1720 \mathrm{veh} / \mathrm{h} & \text { and } & e_{\mu}=7.73 \\
\partial f_{\mathrm{T}} / \partial \sigma=960 \mathrm{veh} / \mathrm{h} & \text { and } & e_{\sigma}=0.662 .
\end{array}
$$

The most sensitive factors here are the median $\operatorname{VoT}, \exp (\mu)$, and the travel time difference, $\Delta T=T_{\mathrm{F}}-T_{\mathrm{T}}$ : a $10 \%$ bias on the travel time difference would induce a bias of almost $20 \%$ on the traffic and revenue!

Error analysis. In order to explain the distribution of output error certain assumptions need to be made about the distribution of exogenous variables:

i) the exogenous errors $\varepsilon_{T_{\mathrm{F}}}$ and $\varepsilon_{T_{\mathrm{T}}}$ which affect the journey times $T_{\mathrm{F}}$ and $T_{\mathrm{T}}$ have centred gaussian distributions with respective variances $\mathrm{s}_{T_{\mathrm{F}}}^{2}$ and $\mathrm{s}_{T_{\mathrm{T}}}^{2}$, and are independent of each other and other errors.

ii) the exogenous error $\varepsilon_{q}$ affecting the level of demand $q$, has a centred gaussian distribution with variance $\mathrm{s}_{q}^{2}$ and is independent of other exogenous errors.

iii) the exogenous errors $\varepsilon_{\mu}$ and $\varepsilon_{\sigma}$ which affect the parameters $\mu$ and $\sigma$ of the VoT distribution, have centred, gaussian distributions with respective variances $\mathrm{s}_{\mu}^{2}$ and $\mathrm{s}_{\sigma}^{2}$. These errors are independent of other exogenous errors, but correlated with each other with covariance $\operatorname{Cov}\left(\varepsilon_{\mu} ; \varepsilon_{\sigma}\right)$. 
In view of (3) and (4), the propagated exogenous error affecting $f_{\mathrm{T}}$ is gaussian and centred, with variance as follows:

$$
\mathrm{s}_{f_{\mathrm{T}}}^{2}=\left(\frac{\partial f_{\mathrm{T}}}{\partial T_{\mathrm{F}}}\right)^{2}\left(\mathrm{~s}_{T_{\mathrm{F}}}^{2}+\mathrm{s}_{T_{\mathrm{T}}}^{2}\right)+\left(\frac{\partial f_{\mathrm{T}}}{\partial q}\right)^{2} \mathrm{~s}_{q}^{2}+\left(\frac{\partial f_{\mathrm{T}}}{\partial \mu}\right)^{2} \mathrm{~s}_{\mu}^{2}+\left(\frac{\partial f_{\mathrm{T}}}{\partial \sigma}\right)^{2} \mathrm{~s}_{\sigma}^{2}+2 \frac{\partial f_{\mathrm{T}}}{\partial \mu} \frac{\partial f_{\mathrm{T}}}{\partial \sigma} \operatorname{Cov}\left(\varepsilon_{\mu} ; \varepsilon_{\sigma}\right) .
$$

Numerical illustration. Based on Leurent (1996b), we shall assume that $\mathrm{s}_{T_{\mathrm{T}}} / T_{\mathrm{T}}=\mathrm{s}_{T_{\mathrm{F}}} / T_{\mathrm{F}}=\mathrm{s}_{q} / q=10 \%, \mathrm{~s}_{\mu} / \mu=\mathrm{s}_{\sigma} / \sigma=4 \%$ and $\operatorname{Cov}\left(\varepsilon_{\mu} ; \varepsilon_{\sigma}\right) / \mu \sigma=-1 \%$. We obtain that $\mathrm{s}_{f_{\mathrm{T}}} / f_{\mathrm{T}}=\mathrm{s}_{\text {Revenue }} /$ Revenue $=37 \%$. The uncertainty which affects the output is at least twice that affecting each of the exogenous variables! This result is, of course, specific to the numerical values which we have used.

Figure 1 shows change in revenue $\mathrm{R}_{\text {dual }}$ in relation to the toll $\mathrm{P}$. We drew the $95 \%$ confidence interval on the average curve $R_{\text {dual }}(P)$. For each value of $P$ the interval bandwidth was divided into three parts corresponding to each of the components in the exogenous error, proportionally to its contribution to the total propagated variance. At low values of $\mathrm{P}$, the trip rate error (propagated as $\frac{\partial f_{\mathrm{T}}}{\partial q} \varepsilon_{q}$ ) plays a major contribution whereas at high values of $\mathrm{P}$ the travel time error (propagated as $\frac{\partial f_{\mathrm{T}}}{\partial T_{\mathrm{F}}}\left(\varepsilon_{T_{\mathrm{F}}}-\varepsilon_{T_{\mathrm{T}}}\right)$ ) is prevailing over it. In all cases the uncertainty in the VoT parameters (propagated as $\frac{\partial f_{\mathrm{T}}}{\partial \mu} \varepsilon_{\mu}+\frac{\partial f_{\mathrm{T}}}{\partial \sigma} \varepsilon_{\sigma}$ ) has a limited contribution: this is because first the whole spectrum of the value of time is considered and second there is a negative correlation between the two parameters $\mu$ and $\sigma$.

It is apparent that the average curve $\mathrm{R}_{\text {single }}(\mathrm{P})$ of the model with a single VoT equal to the mean of $\mathrm{H}$ falls outside the $95 \%$ confidence interval except at very low values of $\mathrm{P}$ : at low, not too small values it is well above the interval, while at high values it is well under it! Thus we are enticed to reject the single criterion model.

\subsection{Model with variable times and fixed demand}

Leurent (1993a) has provided a finite-dimensional formulation for the dual criteria assignment model with variable times. In the case of a two arc network with a fixed volume of demand, the equilibrium state of the model is given by the solution of the following variational inequality defined on $\mathbf{K}_{q}=\left\{\mathbf{f}=\left(f_{\mathrm{F}} ; f_{\mathrm{T}}\right) \geq \mathbf{0} ; f_{\mathrm{F}}+f_{\mathrm{T}}=q\right\}$ :

$$
\text { "find } \mathbf{f} \in \mathbf{K}_{q} \text { such that } \forall \mathbf{g} \in \mathbf{K}_{q},{ }^{t} \mathbf{V}(\mathbf{f}) .(\mathbf{g}-\mathbf{f}) \geq 0 \text { " }
$$

where the components of the mapping $\mathbf{V}$ are defined by $V_{\mathrm{F}}(\mathbf{f})=\mathrm{t}_{\mathrm{F}}(\mathbf{f})-\mathrm{P} / \mathrm{H}^{-1}\left(f_{\mathrm{F}} / q\right)$ and $V_{\mathrm{T}}(\mathbf{f})=\mathrm{t}_{\mathrm{T}}(\mathbf{f})$ and differ from the journey time functions solely by a corrective term in $V_{\mathrm{F}}$. If $\mathrm{P}=0$ then (8) reduces to the well-known variational inequality for Beckmann's model with no tolls (Smith, 1979). 
To complete the definition of $V_{\mathrm{F}}$, let $\eta^{\prime}(\alpha)=1 / \mathrm{H}^{-1}(\alpha)$ be a function on [0;1] onto $\Re$, where the function $\mathrm{H}^{-1}(\alpha)=\max \{v ; \mathrm{H}(v)<\alpha\}$ is the inverse of $\mathrm{H}^{-\mathrm{H}^{-1}}$ is well defined and positive on $] 0 ; 1[$ because $\mathrm{H}$ is (weakly) increasing. At points 0 and 1 we may have to extend the definition of $\eta^{\prime}$. If $\mathrm{H}^{-1}(0)>0$ then $\eta^{\prime}(0)$ is well-defined, but if $\mathrm{H}^{-1}(0)=0$ we define $\eta^{\prime}(0)$ as $+\infty$; looking back at $(8)$, the condition $\mathrm{H}^{-1}(0)=0$ implies that there are very small values of time in the distribution and therefore that the free path must be loaded at equilibrium. At point 1 , if $\mathrm{H}^{-1}(1)<+\infty$ then $\eta^{\prime}(1)$ is welldefined, else we define it as 0 .

By associating a multiplier $\lambda$ to the constraint $f_{\mathrm{F}}+f_{\mathrm{T}}=q$, at equilibrium it holds that if both paths are used then $V_{\mathrm{F}}-\lambda=V_{\mathrm{T}}-\lambda$ or equivalently $V_{\mathrm{F}}=V_{\mathrm{T}}$ and furthermore:

$$
\mathrm{H}^{-1}\left(f_{\mathrm{F}} / q\right)=\mathrm{P} /\left(\mathrm{t}_{\mathrm{F}}(\mathbf{f})-\mathrm{t}_{\mathrm{T}}(\mathbf{f})\right),
$$

which corresponds precisely to $(1)$ as $\mathrm{H}^{-1}\left(f_{\mathrm{F}} / q\right)=\widehat{v}$.

The finite dimension formulation has the advantage of eliminating the need to consider each value of time as a problem variable: only the cut-off VoT, $\hat{v}$, is used and it is taken into account by means of a path flow variable using the transformation $f_{\mathrm{F}} / q=\mathrm{H}(\widehat{v})$. When there are more than two routes, $\overline{\mathrm{R}}$ routes say, these are arranged in order of increasing price and a cut-off VoT is considered for each pair of routes with adjacent prices: all that is required, therefore, is to replicate condition (2) $\bar{R}-1$ times and, in each of the replications, replace the variable $f_{\mathrm{F}}$ by the sum of the flows on routes with prices which are equal to or lower than that of the first route in the pair.

Sensitivity analysis when both paths are loaded, viz. $\left.\alpha \equiv f_{\mathrm{F}} / q \in\right] 0 ; 1[$. Let $\mathrm{Y} \equiv{ }^{t}\left[f_{\mathrm{F}} ; f_{\mathrm{T}} ; \lambda\right]$ be a vector made up of a solution to $(8)$ and the associated multiplier. Let $\mathbf{J} \equiv{ }^{t}\left[V_{\mathrm{F}}-\lambda ; V_{\mathrm{T}}-\lambda ; f_{\mathrm{F}}+f_{\mathrm{T}}-q\right]={ }^{t}\left[\mathrm{t}_{\mathrm{F}}-\mathrm{P} \eta^{\prime}(\alpha)-\lambda ; \mathrm{t}_{\mathrm{T}}-\lambda ; f_{\mathrm{F}}+f_{\mathrm{T}}-q\right]$. By anticipating the outcome of section 2 , the equilibrium solution $\mathrm{Y}$ is a continuously differentiable function of the input vector $\mathrm{X}$ with gradient formulated as:

$$
\nabla_{\mathrm{X}} \mathrm{Y}=-\left(\nabla_{\mathrm{Y}} \mathbf{J}\right)^{-1} \cdot \nabla_{\mathrm{X}} \mathbf{J}
$$

Implementation of this formula requires inversion of the jacobian matrix $\nabla_{\mathrm{Y}} \mathbf{J}$ :

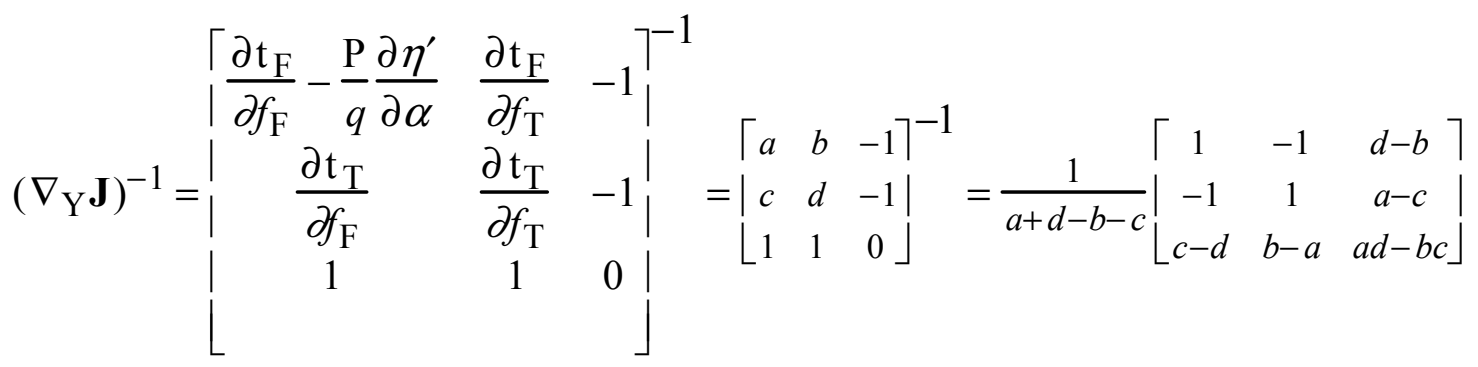




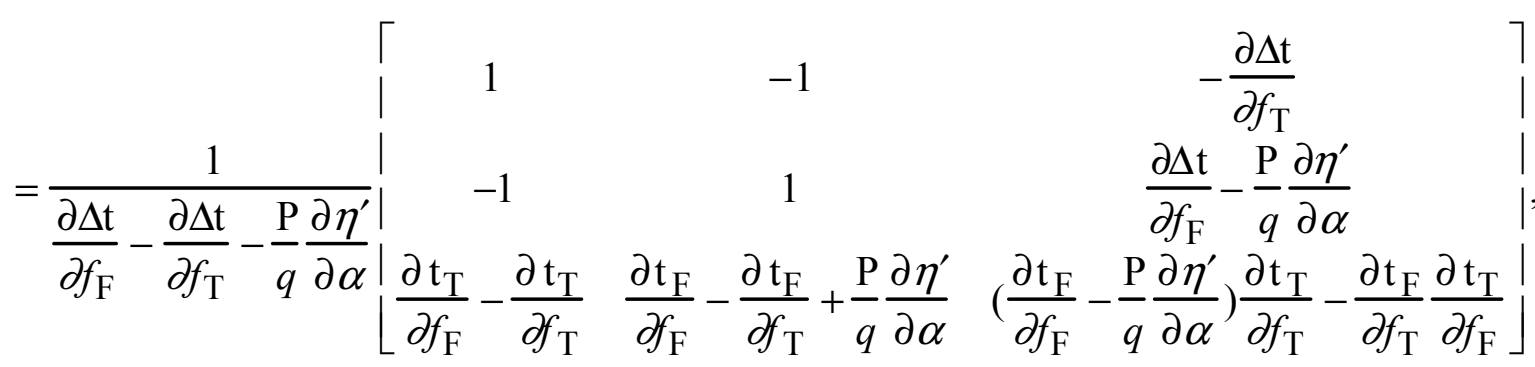

where $\Delta \mathrm{t}=\mathrm{t}_{\mathrm{F}}-\mathrm{t}_{\mathrm{T}}$.

We shall again consider random disturbances on the input vector $\mathrm{X}={ }^{t}\left[T_{\mathrm{F}} ; T_{\mathrm{T}} ; q ; \theta_{i}\right]$. We assume that the travel time function $\mathrm{t}_{\mathrm{F}}\left(\right.$ or $\left.\mathrm{t}_{\mathrm{T}}\right)$ may be decomposed into two parts: a deterministic part which depends on the flow, and a random, flow-independent error which is added to the deterministic part. Our notation $\partial T_{\mathrm{F}}$ refers to a variation in the random part. We obtain:

$$
\begin{aligned}
& \nabla_{\mathrm{X}} \mathbf{J}=\left[\begin{array}{llll}
\frac{\partial \mathbf{J}}{\partial T_{\mathrm{F}}} & \frac{\partial \mathbf{J}}{\partial T_{\mathrm{T}}} & \frac{\partial \mathbf{J}}{\partial q} & \frac{\partial \mathbf{J}}{\partial \theta_{i}}
\end{array}\right]=\left[\begin{array}{cccc}
1 & 0 & \frac{\mathrm{P}}{q} \alpha \frac{\partial \eta^{\prime}}{\partial \alpha} & -\mathrm{P} \frac{\partial \eta^{\prime}}{\partial \theta_{i}}
\end{array}\right]
\end{aligned}
$$

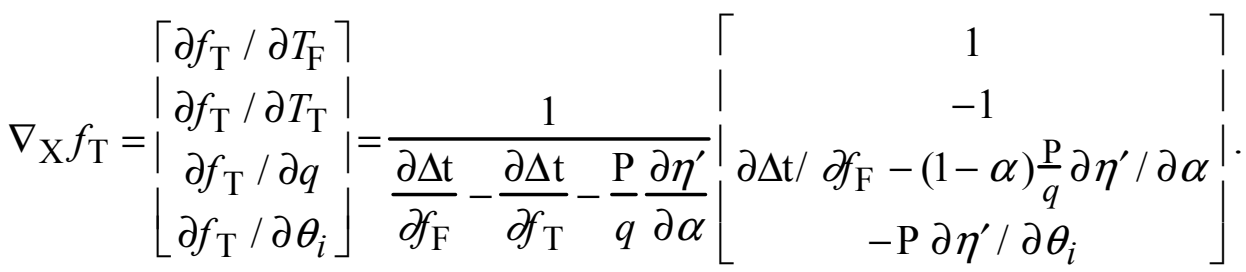

This formula could be obtained in a more straightforward way by using a single problem variable, $f_{\mathrm{T}}$, since in the fixed demand case $f_{\mathrm{F}}$ depends on it via $f_{\mathrm{F}}=q-f_{\mathrm{T}}$. However our treatment serves the two purposes of illustrating that of Section 2 and discussing the influence of each input variable. Let us also compare (13) to $(6)$.

About the travel time disturbances, in both cases the free route time exerts an influence exactly opposite to that of the toll route. If we consider constant travel time functions in $(13 \mathrm{a}, \mathrm{b})$, these conditions reduce to $(6 \mathrm{a}, \mathrm{b})$ since $\partial \eta^{\prime} / \partial \alpha=-1 /\left\{\mathrm{H}^{\prime}(\widehat{v}) \widehat{v}^{2}\right\}$ in which $\widehat{v} \equiv \mathrm{H}^{-1}(\alpha)$. If the travel time functions are not constant, then we intuitively expect that $\partial \Delta \mathrm{t} / \partial f_{\mathrm{F}} \geq 0$ and $\partial \Delta \mathrm{t} / \partial f_{\mathrm{T}} \leq 0$. As $\mathrm{H}$ increases we have that $\partial \eta^{\prime} / \partial \alpha \leq 0$, therefore the denominator in $(13 a, b)$ is larger than that in $(6 a, b)$ : this implies that the sensitivity of the output to the travel times disturbances is reduced in the variable times case.

About the demand volume, $q$, if constant travel times functions are assumed then (13c) reduces to (6c). Otherwise, adding the (expectedly) non negative term $\partial \Delta \mathrm{t} / \partial f_{\mathrm{F}}$ to the 
non negative term $-(1-\alpha) \frac{\mathrm{P}}{q} \partial \eta^{\prime} / \partial \alpha$ makes the absolute value of the numerator in (13c) larger than that in (6c). We know from the argument about the travel times that the denominator is larger. The sensitivity is reduced if and if only the ratio $\left(\partial \Delta \mathrm{t} / \partial f_{\mathrm{F}}\right) /\left(\partial \Delta \mathrm{t} / \partial f_{\mathrm{F}}-\partial \Delta \mathrm{t} / \partial f_{\mathrm{T}}\right)$ is less than $f_{\mathrm{T}} / q$, which depends on the particular numerical values taken by the functions.

About a parameter $\theta_{i}$ in the cumulative distribution function $\mathrm{H}$, we shall address the example of a value of time uniformly distributed on $[0 ; A]$. In this case the CDF is given on $\left[0 ;+\infty\left[\right.\right.$ by $\mathrm{H}(v)=\min (v / A ; 1)$ and its inverse on $[0 ; 1]$ by $\mathrm{H}^{-1}(\alpha)=A \alpha$ : hence $\eta=1 / \alpha A$. Letting $\theta_{i}=A$, (13d) applied to constant travel times functions yields $\partial f_{\mathrm{T}} / \partial A=q\left(\partial \eta^{\prime} / \partial A\right) /\left(\partial \eta^{\prime} / \partial \alpha\right)$ and therefore $\partial f_{\mathrm{T}} / \partial A=q \alpha / A=f_{\mathrm{T}} / A$ which is also derived from $(6 \mathrm{~d})$. If the travel times vary with respect to the flows, the argument about the denominator in (13) still implies that the sensitivity is less than that in the model with fixed travel times.

Numerical application. We used the same example data as in sub-section 1.1, except for the travel times which were assumed to depend on the link flow on the basis of $\mathrm{t}_{k}(\mathbf{f})=t_{k}^{0}\left(1+\gamma_{k}+\left(\left[\alpha_{k} \cdot\left(1-\frac{f_{k}}{N_{k}}\right)\right]^{2}+\beta_{k}{ }^{2}\right)^{1 / 2}-\alpha_{k} \cdot\left(1-\frac{f_{k}}{N_{k}}\right)-\beta_{k}\right)$ where $t_{k}^{0}$ is a freeflow travel time, $N_{k}$ is a practical capacity, $\alpha_{k}$ and $\gamma_{k}\left(>\alpha_{k}\right)$ are parameters from which $\beta_{k}$ is derived based on $\beta_{k}=\gamma_{k}\left(\alpha_{k}-\gamma_{k} / 2\right) /\left(\alpha_{k}-\gamma_{k}\right)$. The parameters were given numerical values of $t_{\mathrm{F}}^{0}=0.30 \mathrm{~h}, N_{\mathrm{F}}=5000 \mathrm{veh} / \mathrm{h}, \alpha_{\mathrm{F}}=2.5$ and $\gamma_{\mathrm{F}}=1.5$ for the free path, and of $t_{\mathrm{T}}^{0}=0.18 \mathrm{~h}, N_{\mathrm{T}}=1000 \mathrm{veh} / \mathrm{h}, \alpha_{\mathrm{T}}=4.0$ and $\gamma_{\mathrm{T}}=0.5$ for the toll path. At point $\mathrm{P}=3 \$$, the journey time difference is still equal to $0.214 \mathrm{~h}$.

Figure 2 shows changes in revenues $R_{\text {dual }}$ and $R_{\text {single }}$ in relation to the toll $P$, based on the same probabilistic assumptions on the exogenous errors and the same principles of representation as in figure 1 . We observe that the contribution of the VoT error remains small, as in figure 1 . Contrary to figure 1 , the relative contributions of the demand volume error and the travel time error are broadly equivalent at all toll levels. At point $\mathrm{P}=3 \$$ where the two dual criteria models differ from each other solely in the propagation coefficients, these are reduced in the variable time case, as expected from the discussion of (13). At last, the difference between the two revenue curves $\mathrm{R}_{\text {dual }}$ and $\mathrm{R}_{\text {single }}$ in the variable times case is much smaller than in the fixed times case, because congestion effects can entail a multi-path assignment even in the case of a single value of time.

\subsection{Model with variable times and elastic demand}

Let us now assume that the volume of demand depends on the level of service, $\bar{S}$, 
which is defined in an aggregate way as the generalized time averaged over the distribution of values of time:

$$
\bar{S} \equiv \int \min \left\{\mathrm{t}_{\mathrm{F}} ; \mathrm{t}_{\mathrm{T}}+\mathrm{P} / v\right\} \mathrm{dH}(v) .
$$

Let $\mathrm{D}$ denote the demand function which relates $q$ to $\bar{S}$ :

$$
q=\mathrm{D}(\bar{S}) .
$$

This dependency is approximation : it is only completely valid if, for each VoT $v$, the density of demand $q_{v}$ for this VoT complies with $q_{v}=\mathrm{D}\left(\min \left\{\mathrm{t}_{\mathrm{F}} ; \mathrm{t}_{\mathrm{T}}+\mathrm{P} / v\right\}\right)$ with a demand function $\mathrm{D}$ which is affine and the same for all values of time.

At last, let us define on the set of all non negative path flows $\mathbf{K} \equiv\left\{\mathbf{f}=\left(f_{\mathrm{F}} ; f_{\mathrm{T}}\right) \geq \mathbf{0}\right\}$ a mapping $\mathbf{W}$ by:

$$
\mathbf{W}(\mathbf{f})=\left[\begin{array}{l}
\left\lceil W_{\mathrm{F}}(\mathbf{f})\right\rceil \\
W_{\mathrm{T}}(\mathbf{f})
\end{array}\right]=\left[\begin{array}{c}
\mathrm{t}_{\mathrm{F}}(\mathbf{f})+\mathrm{P}\left(\eta(1)-\eta(\alpha)-(1-\alpha) \eta^{\prime}(\alpha)\right)-\mathrm{D}^{-1}\left(f_{\mathrm{F}}+f_{\mathrm{T}}\right) \\
\mathrm{t}_{\mathrm{T}}(\mathbf{f})+\mathrm{P}\left(\eta(1)-\eta(\alpha)+\alpha \eta^{\prime}(\alpha)\right)-\mathrm{D}^{-1}\left(f_{\mathrm{F}}+f_{\mathrm{T}}\right)
\end{array}\right],
$$

where $\mathrm{D}^{-1}(q)=\min \{x ; \mathrm{D}(x)<q\}, \alpha=f_{\mathrm{F}} /\left(f_{\mathrm{F}}+f_{\mathrm{T}}\right)$ and $\eta$ is a primitive of $\eta^{\prime}$.

The components of $\mathbf{W}$ are the path travel time functions corrected by terms which capture the distribution of VoT and the variability of the demand volume.

The equilibrium state of the dual criteria model with variable times and variable demand solves the following variational inequality:

$$
\text { "find } \mathbf{f} \in \mathbf{K} \text { such that } \forall \mathbf{g} \in \mathbf{K},{ }^{t} \mathbf{W}(\mathbf{f}) .(\mathbf{g}-\mathbf{f}) \geq 0 \text { ". }
$$

At equilibrium we check that if $\left.\alpha \equiv f_{\mathrm{F}} / q \in\right] 0 ; 1\left[\right.$ then $W_{\mathrm{F}}=W_{\mathrm{T}}=0$ which is equivalent to:

i) $\mathrm{t}_{\mathrm{F}}(\mathbf{f})-\mathrm{P} \eta^{\prime}(\alpha)=\mathrm{t}_{\mathrm{T}}(\mathbf{f})$ which amounts to the same thing as (1),

ii) $\mathrm{D}^{-1}=\alpha\left(W_{\mathrm{F}}+\mathrm{D}^{-1}\right)+(1-\alpha)\left(W_{\mathrm{T}}+\mathrm{D}^{-1}\right)=\int_{0}^{\alpha} \mathrm{t}_{\mathrm{F}} \mathrm{d} \xi+\int_{\alpha}^{1}\left\{\mathrm{t}_{\mathrm{T}}+\mathrm{P} \eta^{\prime}(\xi)\right\} \mathrm{d} \xi$ which, after changing the integration variable from $\xi$ to $v$ on the basis of $\xi=\mathrm{H}(v)$, yields (14) because at equilibrium the minimum generalized time is provided to the VoTs less than $\mathrm{H}^{-1}(\alpha)$ by the free route and to those above this value by the toll route.

Let $\mathrm{Y}={ }^{t}\left[f_{\mathrm{F}} ; f_{\mathrm{T}}\right]$ be a vector consisting of a solution to (17). It is possible once more to apply (10), now with $\mathbf{J}=\mathbf{W}$. Under the assumption that $0<\alpha<1$ and denoting by $\mathrm{D}^{-1 /}$ the partial derivative of $\mathrm{D}^{-1}$ with respect to its explicit argument, $q$, we obtain that: 


$$
\begin{aligned}
& \nabla_{\mathrm{Y}} \mathbf{J}=\left[\begin{array}{ll}
\frac{\partial \mathrm{t}_{\mathrm{F}}}{\partial f_{\mathrm{F}}}-\frac{\mathrm{P}}{q}(1-\alpha)^{2} \frac{\partial \eta^{\prime}}{\partial \alpha}-\mathrm{D}^{-1 /} & \frac{\partial \mathrm{t}_{\mathrm{F}}}{\partial f_{\mathrm{T}}}+\frac{\mathrm{P}}{q} \alpha(1-\alpha) \frac{\partial \eta^{\prime}}{\partial \alpha}-\mathrm{D}^{-1 /} \\
\frac{\partial \mathrm{t}_{\mathrm{T}}}{\partial f_{\mathrm{F}}}+\frac{\mathrm{P}}{q} \alpha(1-\alpha) \frac{\partial \eta^{\prime}}{\partial \alpha}-\mathrm{D}^{-1 /} & \frac{\partial \mathrm{t}_{\mathrm{T}}}{\partial f_{\mathrm{T}}}-\frac{\mathrm{P}}{q} \alpha^{2} \frac{\partial \eta^{\prime}}{\partial \alpha}-\mathrm{D}^{-1 /}
\end{array}\right], \\
& \left.\left(\nabla_{\mathrm{Y}} \mathbf{J}\right)^{-1}=\frac{1}{\operatorname{Det}} \mid \begin{array}{cc}
\frac{\partial \mathrm{t}_{\mathrm{T}}}{\partial f_{\mathrm{T}}}-\frac{\mathrm{P}}{q} \alpha^{2} \frac{\partial \eta^{\prime}}{\partial \alpha}-\mathrm{D}^{-1 /} & -\frac{\partial \mathrm{t}_{\mathrm{F}}}{\partial f_{\mathrm{T}}}-\frac{\mathrm{P}}{q} \alpha(1-\alpha) \frac{\partial \eta^{\prime}}{\partial \alpha}+\mathrm{D}^{-1 /} \\
-\frac{\partial \mathrm{t}_{\mathrm{T}}}{\partial f_{\mathrm{F}}}-\frac{\mathrm{P}}{q} \alpha(1-\alpha) \frac{\partial \eta^{\prime}}{\partial \alpha}+\mathrm{D}^{-1 /} & \frac{\partial \mathrm{t}_{\mathrm{F}}}{\partial f_{\mathrm{F}}}-\frac{\mathrm{P}}{q}(1-\alpha)^{2} \frac{\partial \eta^{\prime}}{\partial \alpha}-\mathrm{D}^{-1 /}
\end{array}\right]
\end{aligned}
$$

where Det $=\left|\nabla_{\mathrm{Y}} \mathbf{J}\right|=\frac{\partial \mathrm{t}_{\mathrm{F}}}{\partial f_{\mathrm{F}}} \frac{\partial \mathrm{t}_{\mathrm{T}}}{\partial f_{\mathrm{T}}}-\frac{\partial \mathrm{t}_{\mathrm{T}}}{\partial f_{\mathrm{F}}} \frac{\partial \mathrm{t}_{\mathrm{F}}}{\partial f_{\mathrm{T}}}-\mathrm{D}^{-1 /} \cdot\left(\frac{\partial \Delta \mathrm{t}}{\partial f_{\mathrm{F}}}-\frac{\partial \Delta \mathrm{t}}{\partial f_{\mathrm{T}}}-\frac{\mathrm{P}}{q} \frac{\partial \eta^{\prime}}{\partial \alpha}\right)$

$$
-\frac{\mathrm{P}}{q} \frac{\partial \eta^{\prime}}{\partial \alpha}\left(\alpha^{2} \frac{\partial \mathrm{t}_{\mathrm{F}}}{\partial f_{\mathrm{F}}}+(1-\alpha)^{2} \frac{\partial \mathrm{t}_{\mathrm{T}}}{\partial f_{\mathrm{T}}}+\alpha(1-\alpha)\left(\frac{\partial \mathrm{t}_{\mathrm{T}}}{\partial f_{\mathrm{F}}}+\frac{\partial \mathrm{t}_{\mathrm{F}}}{\partial f_{\mathrm{T}}}\right)\right),
$$

by again using the expression $\Delta \mathrm{t}=\mathrm{t}_{\mathrm{F}}-\mathrm{t}_{\mathrm{T}}$.

Sensitivity analysis. We shall consider the input vector $\mathrm{X}={ }^{t}\left[T_{\mathrm{F}} ; T_{\mathrm{T}} ; q_{0} ; \theta_{i}\right]$, in which $q_{0}$ is a parameter in the demand function $\mathrm{D}$ (and therefore in the inverse demand function $\mathrm{D}^{-1}$ ). We obtain:

$\nabla_{\mathrm{X}} \mathbf{J}=\left[\begin{array}{llll}\frac{\partial \mathbf{J}}{\partial T_{\mathrm{F}}} & \frac{\partial \mathbf{J}}{\partial T_{\mathrm{T}}} & \frac{\partial \mathbf{J}}{\partial q_{0}} & \frac{\partial \mathbf{J}}{\partial \theta_{i}}\end{array}\right]=\left[\begin{array}{llll}1 & 0 & -\frac{\partial \mathrm{D}^{-1}}{\partial q_{0}} & \mathrm{P}\left[\frac{\partial \eta(1)}{\partial \theta_{i}}-\frac{\partial \eta(\alpha)}{\partial \theta_{i}}-(1-\alpha) \frac{\partial \eta^{\prime}(\alpha)}{\partial \theta_{i}}\right]\end{array}\right]$,

$$
\begin{aligned}
& \left\lceil\frac{\partial f_{\mathrm{T}}}{\partial T_{\mathrm{F}}}\right\rceil \quad \frac{\partial \mathrm{t}_{\mathrm{T}}}{\partial f_{\mathrm{F}}}+\frac{\mathrm{P}}{q} \alpha(1-\alpha) \frac{\partial \eta^{\prime}}{\partial \alpha}-\mathrm{D}^{-1 /} \\
& \left|\frac{\partial f_{\mathrm{T}}}{\partial T_{\mathrm{T}}}\right|_{1} \mid \quad-\frac{\partial \mathrm{t}_{\mathrm{F}}}{\partial f_{\mathrm{F}}}+\frac{\mathrm{P}}{q}(1-\alpha)^{2} \frac{\partial \eta^{\prime}}{\partial \alpha}+\mathrm{D}^{-1 /} \\
& \left|\frac{\partial f_{\mathrm{T}}}{\partial q_{0}}\right|=\frac{1}{\mathrm{Det}} \mid \quad\left(\frac{\partial \Delta \mathrm{t}}{\partial f_{\mathrm{F}}}-(1-\alpha) \frac{\mathrm{P}}{q} \frac{\partial \eta^{\prime}}{\partial \alpha}\right) \frac{\partial \mathrm{D}^{-1}}{\partial q_{0}} \\
& \left\lfloor\frac{\partial f_{\mathrm{T}}}{\partial \theta_{i}}\right\rfloor \quad\left|-\mathrm{P}\left(\left(\frac{\partial \eta(1)}{\partial \theta_{i}}-\frac{\partial \eta(\alpha)}{\partial \theta_{i}}\right)\left(\frac{\partial \Delta \mathrm{t}}{\partial f_{\mathrm{F}}}-\frac{\mathrm{P}}{q}(1-\alpha) \frac{\partial \eta^{\prime}}{\partial \alpha}\right)+\frac{\partial \eta^{\prime}}{\partial \theta_{i}}\left(\frac{\partial \mathrm{t}_{\mathrm{F}}}{\partial f_{\mathrm{F}}}+\alpha \frac{\partial \Delta \mathrm{t}}{\partial f_{\mathrm{F}}}-\mathrm{D}^{-1 /}\right)\right)\right|
\end{aligned}
$$

The addition of a demand-supply equilibrium mechanism (15) entails a stronger coupling of the inputs in the propagation formulae. While in (6) and (13) their joint effects were mainly additive, in (20) there are multiplicative as well as additive effects.

Let us show that (20) reduces to (13) in the fixed demand case by considering a demand function with constant elasticity to the average generalized travel time, viz. $q=\mathrm{D}(\bar{S})=q_{0}\left(\bar{S} / S_{0}\right)^{e_{0}}$, and letting $e_{0} \rightarrow 0^{-}$to simulate a fixed demand volume. As $\mathrm{D}^{-1}(q)=S_{0}\left(q / q_{0}\right)^{1 / e_{0}}$ and $\mathrm{D}^{-1 /}(q)=\bar{S} / e_{0} q$, if $e_{0} \rightarrow 0^{-}$then $\mathrm{D}^{-1 /} \rightarrow-\infty$ which 
makes it prevail over any finite term to which it is added. Thus Det reduces to the term which contains $\mathrm{D}^{-1 /}$, and in a straightforward way (20a), (20b) and (20d) reduce respectively to (13a), (13b) and (13d). (20c) reduces to (13c) multiplied by $q / q_{0}$ because $\partial \mathrm{D}^{-1} / \partial q_{0}=-q \mathrm{D}^{-1 /} / q_{0}:$ as $e_{0} \rightarrow 0^{-}$we have that $q / q_{0} \approx 1$.

Under the intuitive assumptions that the travel time functions do not decrease with respect to any flow and that $\partial \mathrm{t}_{\mathrm{F}} / \partial f_{\mathrm{F}} \geq \partial \mathrm{t}_{\mathrm{T}} / \partial f_{\mathrm{F}}$ and $\partial \mathrm{t}_{\mathrm{T}} / \partial f_{\mathrm{T}} \geq \partial \mathrm{t}_{\mathrm{F}} / \partial f_{\mathrm{T}}$, Det is the sum of three non negative terms which are: the difference of two products of travel time derivatives, a second term which contains $\mathrm{D}^{-1 /}$ and a third which is a product of the toll by derivatives of travel time functions. Then Det is larger than its own second term. Therefore, at point $q=q_{0}$, the sensitivity to $q_{0}$ of the model with constant elasticity variable demand is less than that to $q$ in the fixed demand case. Similarly the sensitivity to the travel time on the toll route decreases: the numerator of (20b) has two non negative terms that balance the non positive $\mathrm{D}^{-1 /}$ and reduce the absolute value, while the absolute value of the denominator is increased.

Numerical application. We used the same example data as in sub-section 1.2, except for the demand volume which was assumed to depend on the mean generalized travel time with a fixed elasticity $e_{0}=-0.6$ and to take the value $q_{0}=3000 \mathrm{veh} / \mathrm{h}$ at point $S_{0}=0.413 \mathrm{~h}$. Therefore at point $\mathrm{P}=3 \$$ the application data are the same as in subsections 1.1 and 1.2, except for the propagation coefficients.

Figure 3 shows changes in revenues $R_{\text {dual }}$ and $R_{\text {single }}$ in relation to the toll $P$, based on the same probabilistic assumptions on the exogenous errors and the same principles of representation as in figure 1 . We observe that the exogenous error on the VoT error has a slightly more important contribution than in figures 1 and 2. As in figure 2 the contribution of the travel time error is broadly equivalent to that of the demand volume disturbance; however their overall effect is less than in the fixed demand case, as was expected from the discussion of (20). In figures 2 and 3, the single criterion models underestimate the revenue at all positive toll levels: while of little importance at low toll values, this is largely unrealistic at medium and high values.

\section{THE GENERAL CASE}

Section 1 contained a full analytical treatment in order to propagate the exogenous error through the dual criteria assignment model, in the case of a two arc network. By progressively adding behavioural features to the model, we have observed that enriching the behavioural contents of the model provides a means of reducing the exogenous error propagated in the output. In the general case of an assignment problem of any given size, it is possible to use the same propagation formulae, but these cannot 
be solved in a closed form as in the case of a two arc network. We shall state these for the dual criteria assignment model with continuously distributed values of time, elastic demand, travel times which depend on flow and side constraints.

Sub-sections 2.1 and 2.2 review the assumptions in the dual criteria model and the mathematical characterization given by Leurent (1996a). In sub-section 2.3, an alternative characterization is derived in order to avail ourselves of the framework for sensitivity analysis worked out by Tobin (1986) and Tobin and Friesz (1988). The assumptions and results which relate to the framework are restated in sub-section 2.4. Sub-section 2.5 contains the formulae required to conduct the sensitivity analysis of the dual criteria model in the case of a general network.

\subsection{Basic assumptions of the model}

We shall use the notation which follows. Let $i$ be an origin-destination (O-D) pair with trip rate $q_{i}$ and demand function $\mathrm{D}_{i}\left(\bar{S}_{i}\right)$, where $\bar{S}_{i}$ is a generalized travel time to be defined later. $\mathrm{H}_{i}$ is the cumulative distribution function for the VoT $v$ of the trips on the O-D pair: thus $\operatorname{Pr}(\{v \leq x\})=\mathrm{H}_{i}(x)$ for these trips. Let $\eta_{i}^{\prime}(\alpha)=1 / \mathrm{H}_{i}^{-1}(\alpha)$ for every $\alpha$ in $[0 ; 1]$ : at points 0 and 1 the definition of $\eta_{i}^{\prime}$ may need extension as in sub-section 1.2. Let $\eta_{i}$ be a primitive of $\eta_{i}^{\prime}$.

Let $k$ be a path on the O-D pair $i$, with flow $f_{i k}$, travel time $\mathrm{T}_{i k}$ and travel price $\mathrm{P}_{i k}$. It holds that $q_{i}=\sum_{k \in i} f_{i k}$. To a trip with VoT $v$, the generalized time of travel on path $k$ is $\mathrm{G}_{i k}(v) \equiv \mathrm{T}_{i k}+\mathrm{P}_{i k} / v$. Each trip-maker is assumed to choose a path $k$ with a minimum generalized travel time with respect to his VoT (the path is said efficient for this VoT). Thus the mean minimum generalized travel time is $\bar{S}_{i} \equiv \int\left\{\min _{k \in i} \mathrm{G}_{i k}(v)\right\} \mathrm{dH}_{i}(v)$ which is used as the input to the demand function.

The vector $\mathbf{f}=\left[f_{i k}\right]_{i k}$ is called the path flow pattern.

Let $a$ be a network link (= arc) with flow $x_{a}$. Let a link path incidence indicator $\delta_{i k}^{a} \equiv 1$ if link $a$ is incident to path $k$ or equal to 0 if it is not. Therefore $x_{a}=\sum_{i k} \delta_{i k}^{a} f_{i k}$.

We shall assume that the travel time on link $a$ depends on the path flow pattern $\mathbf{f}$ based on a link travel time function $\mathrm{t}_{\alpha}(\mathbf{f})$. We shall also assume that the flow is subject to conditions $\mathbf{z}_{b}(\mathbf{f}) \leq 0$ called side constraints (Larsson and Patriksson, 1994). If such a side constraint is binding i.e. $\mathbf{z}_{b}(\mathbf{f})=0$, then, linking a multiplier $w_{b} \geq 0$ to this because of the network conditions, we add a penalty $w_{b} \partial \mathrm{z}_{b} / \partial f_{i k}$ to the travel time for each path $k$. For instance, we may define a local capacity constraint $x_{a}(\mathbf{f})-C_{a} \leq 0$ for link $a$; if it is binding then the travel time of a path $k$ will be penalized by $w_{a} \partial\left(x_{a}-C_{a}\right) / \partial f_{i k}=w_{a} \delta_{i k}^{a}$. Thus, the travel time of a path $k$ is given by the following 
formula $\mathrm{T}_{i k}=\left(\sum_{a} \delta_{i k}^{a} \mathrm{t}_{a}\right)+\left(\sum_{b} w_{b} \partial \mathrm{z}_{b} / \partial f_{i k}\right)$

\subsection{Definition and characterization of a dual criteria equilibrium}

The continuous distribution of the VoT generates an infinity of distinct behaviors. However the problem may be redefined with path flow variables, hence with a finite number of variables, by linking implicitly each VoT to a path that is efficient for it. This is performed by selecting the efficient paths by means of conditions on the isoprice classes of paths, which are defined next.

Isoprice Classes of Paths. For every O-D pair $i$, we divide the paths into the equivalency classes of the following equivalency relationship $\mathrm{R}_{i}: k \mathrm{R}_{i} k^{\prime}$ iff $\mathrm{P}_{i k}=\mathrm{P}_{i k^{\prime}}$. We call these isoprice classes of paths.

We index the isoprice classes with respect to increasing prices from 1 to $\bar{m}_{i}$. We shall define $\mathrm{M}_{i}(k)$ as the class index of the path $(i) k$. Let $\overline{\mathrm{P}}_{i m}$ be the price on the path(s) of the $m$-th class and $\overline{\mathrm{T}}_{i m}$ be the minimum travel time proper across the paths of this class. Let $q_{i n}(\mathbf{f}) \equiv \sum_{k ; \mathrm{M}_{i}(k)=n} f_{i k}$ be the traffic flow on the paths of the $n$-th isoprice class, and $q_{i m}^{\prime} \equiv \sum_{n \leq m} q_{i n}$ the traffic flow on the paths whose prices are less than or equal to those of the $m$-th class. It also holds that $q_{i}=\sum_{n} q_{i n}=q_{i \bar{m}_{i}}^{\prime}$. We define $q_{i 0}=q_{i 0}^{\prime} \equiv 0$.

Definition of a dual criteria equilibrium. A dual criteria equilibrium is a bi-tuple $(\mathbf{f} ; \mathbf{w})$ where $\mathbf{f}$ is a vector of path flows $f_{i k} \geq 0$ and $\mathbf{w}$ is a vector of the multipliers $w_{b}$ related to the side constraints $\mathrm{z}_{b}(\mathbf{f}) \leq 0$, such that:

(i) Formation of the travel times: $\mathrm{T}_{i k}(\mathbf{f} ; \mathbf{w})=\left(\sum_{a} \delta_{i k}^{a} \mathrm{t}_{a}(\mathbf{f})\right)+\left(\sum_{b} w_{b} \frac{\partial \mathrm{z}_{b}(\mathbf{f})}{\partial f_{i k}}\right)$ with $w_{b} \geq 0, \mathrm{z}_{b}(\mathbf{f}) \leq 0$ and $w_{b} \mathrm{z}_{b}(\mathbf{f})=0$.

(ii) Supply-demand exchange: $q_{i}(\mathbf{f}) \equiv \sum_{k} f_{i k}=\mathrm{D}_{i}\left(\int\left\{\min _{k} \mathrm{G}_{i k}(v)\right\} \mathrm{dH}_{i}(v)\right)$.

(iii) Optimizing behaviour of the trip-makers: for each O-D pair $i$, within each isoprice class $m$ it holds that $\forall k \in m, f_{i k}>0 \Rightarrow \mathrm{T}_{i k}=\min _{k \in m} \mathrm{~T}_{i k} \equiv \overline{\mathrm{T}}_{i m}$, and between the isoprice classes the class flow pattern $\left[q_{i m}(\mathbf{f})\right]_{m}$ satisfies for each class $n$ that $q_{\text {in }}=q_{i} \int_{\mathrm{E}_{\text {in }}} \mathrm{dH}_{i}(v)$, where $\mathrm{E}_{\text {in }}$ is the set of the VoTs for which class $n$ is efficient based on the supply conditions $\left\{\left(\overline{\mathrm{P}}_{i m} ; \overline{\mathrm{T}}_{i m}\right)\right\}_{m}$.

The last condition states that the infinitesimal flow carried by every value of time is assigned to an isoprice class that supplies it with a minimum class generalized time (part between), and that within the class it is assigned to a path with least travel time.

The path impedance function of path $k$ is defined as 


$$
\begin{aligned}
& \mathrm{I}_{i k}(\mathbf{f} ; \mathbf{w}) \equiv \mathrm{T}_{i k}+\sum_{n=\mathrm{M}_{i}(k)}^{\bar{m}_{i}-1}\left(\overline{\mathrm{P}}_{i, n}-\overline{\mathrm{P}}_{i, n+1}\right) \eta_{i}^{\prime}\left(\frac{q_{\text {in }}^{\prime}}{q_{i}}\right) \\
& \quad-\mathrm{D}_{i}^{-1}\left(q_{i}\right)+\overline{\mathrm{P}}_{i \bar{m}_{i}} \eta_{i}(1)-\overline{\mathrm{P}}_{i 1} \eta_{i}(0)+\sum_{n=1}^{\bar{m}_{i}-1}\left(\overline{\mathrm{P}}_{i, n}-\overline{\mathrm{P}}_{i, n+1}\right)\left(\eta_{i}\left(\frac{q_{\text {in }}^{\prime}}{q_{i}}\right)-\frac{q_{\text {in }}^{\prime}}{q_{i}} \cdot \eta_{i}^{\prime}\left(\frac{q_{\text {in }}^{\prime}}{q_{i}}\right)\right),
\end{aligned}
$$

which depends on $(\mathbf{f} ; \mathbf{w})$ through $\mathrm{T}_{i k}$ and the $q_{\text {in }}^{\prime}$. On an O-D pair with a single path, (21) is reduced to $\mathrm{T}+\mathrm{P}(\eta(1)-\not(0))-\mathrm{D}^{-1}(q)$ as in Beckmann's model with a single VoT $v=1 /(\eta(1)-\eta(0))$. In the unconstrained case, when there are only two paths, (21) is reduced to either $W_{\mathrm{F}}$ or $W_{\mathrm{T}}$ of sub-section 1.3.

Let us now define the mapping $\mathbf{V}$ on $\left(\mathfrak{R}^{+}\right)^{\mathrm{N}}$ onto $\mathfrak{R}^{\mathrm{N}}$ (where $\mathrm{N}$ sums up the dimensions of $\mathbf{f}$ and $\mathbf{w})$ by $(\mathbf{f} ; \mathbf{w}) \mapsto \mathbf{V}(\mathbf{f} ; \mathbf{w})={ }^{t}\left[{ }^{t}\left[\mathrm{I}_{i k}(\mathbf{f} ; \mathbf{w})\right]_{i k} ;{ }^{t}\left[-\mathrm{z}_{b}(\mathbf{f})\right]_{b}\right]$. It is shown in Leurent (1996a) that $\left(\mathbf{f}^{*} ; \mathbf{w}^{*}\right) \geq \mathbf{0}$ is a dual criteria equilibrium if and only if it solves the following variational inequality (22):

"find $\left(\mathbf{f}^{*} ; \mathbf{w}^{*}\right) \geq \mathbf{0}$ such that $\forall(\mathbf{f} ; \mathbf{w}) \geq \mathbf{0},{ }^{t} \mathbf{V}\left(\mathbf{f}^{*} ; \mathbf{w}^{*}\right) \cdot\left(\mathbf{f}-\mathbf{f}^{*} ; \mathbf{w}-\mathbf{w}^{*}\right) \geq 0$ ".

\subsection{Additional assumptions and modified characterization}

In order to extend the results of Tobin and Friesz (1988) to the dual criteria model, we shall modify and add to the assumptions of the problem (22) so that only flow variables f are present and also in order to consider affine linear constraints $d_{\ell}(\mathbf{f})=0$ for values of $\ell$ between 1 and $\mathrm{N}_{\mathrm{d}}$, which, in particular, allow us to fix the demand volume for certain origin-destination pairs. Then, in the definition of a dual criteria equilibrium, each path travel time $\mathrm{T}_{i k}$ is augmented by a term $-\sum_{\ell} \lambda_{\ell} \partial \mathrm{d}_{\ell} / \partial f_{i k}$ where $\lambda_{\ell}$ is the multiplier associated with the constraint $\mathrm{d}_{\ell}(\mathbf{f})=0$. If a such constraint is used to fix an O-D volume, the travel time differences between the paths of the O-D pair are not modified.

We shall now assume the following:

i) that the functions $z_{b}$ are convex and differentiable (this applies particularly to capacity constraints of the type $\left.x_{a}-C_{a} \leq 0\right)$,

ii) that there are affine linear constraints $d_{\ell}(\mathbf{f})=0$,

iii) that the gradients $\nabla \mathrm{z}_{b}(\mathbf{f})$ and $\nabla \mathrm{d}_{\ell}(\mathbf{f})$ are independent of each other.

Let $\mathbf{K}=\left\{\mathbf{f} \geq \mathbf{0} ; \mathrm{z}_{b}(\mathbf{f}) \leq 0 \forall b \in 1 . . \mathrm{N}_{\mathrm{b}}\right.$ and $\left.\mathrm{d}_{\ell}(\mathbf{f})=0 \forall \ell \in 1 . . \mathrm{N}_{\mathrm{d}}\right\}$ and let $\mathbf{U}$ be the mapping defined on $\mathbf{K}$ onto $\mathfrak{R}^{\mathrm{N}_{k}}$ where $\mathrm{N}_{k}$ is the number of paths, such that $\mathbf{U}(\mathbf{f}) \equiv\left[\mathrm{I}_{i k}(\mathbf{f} ; \mathbf{0})\right]_{i k}$. The following theorem is proven in Leurent (1997).

Theorem 1. A vector of flow per path $\mathbf{f}^{*} \geq \mathbf{0}$ is involved in a dual criteria equilibrium under the constraints $\mathrm{z}_{b}(\mathbf{f}) \leq 0$ and $\mathrm{d}_{\ell}(\mathbf{f})=0$ if and only if it resolves the following 
variational inequality (23):

$$
\text { "find } \mathbf{f}^{*} \in \mathbf{K} \text { such that } \forall \mathbf{f} \in \mathbf{K},{ }^{t} \mathbf{U}\left(\mathbf{f}^{*}\right) .\left(\mathbf{f}-\mathbf{f}^{*}\right) \geq 0 \text {." }
$$

\subsection{The results of Tobin and Friesz (1988)}

We shall restate for completeness the results of Tobin (1986) which are useful in order to analyze the sensitivity of a solution to a finite-dimensional variational inequality.

The set of all possible model input combinations is $\mathbf{K}=\left\{\mathbf{x} \in \mathfrak{R}^{n} ; \mathbf{g}(\mathbf{x}) \geq \mathbf{0}, \mathbf{d}(\mathbf{x})=\mathbf{0}\right\}$, where $\mathbf{g}$ is a differentiable mapping on $\Re^{n}$ onto $\Re^{m}$ and $\mathbf{d}$ is a linear affine mapping on $\Re^{n}$ onto $\Re^{p}$. We shall assume that any equilibrium state $\mathbf{x}^{*}$ of the model solves the following variational inequality, where $\mathbf{F}$ is a continuous mapping on $\mathbf{K}$ onto $\mathfrak{R}^{n}$ :

$$
\text { "find } \mathbf{x}^{*} \in \mathbf{K} \text {, such that } \forall \mathbf{x} \in \mathbf{K},{ }^{t} \mathbf{F}\left(\mathbf{x}^{*}\right) .\left(\mathbf{x}-\mathbf{x}^{*}\right) \geq \mathbf{0} \text {." }
$$

Let $\boldsymbol{\varepsilon}$ be a disturbance vector standing for the exogenous error: we shall write $\mathbf{F}(\mathbf{x} \mid \boldsymbol{\varepsilon})$, $\mathbf{g}(\mathbf{x} \mid \boldsymbol{\varepsilon})$ and $\mathbf{d}(\mathbf{x} \mid \boldsymbol{\varepsilon})$ to take $\boldsymbol{\varepsilon}$ into account. Let also $\mathbf{y}={ }^{t}\left[{ }^{t} \mathbf{x} ;{ }^{t} \pi ;{ }^{t} \lambda\right]$ where $\boldsymbol{\pi}$ belongs to $\mathfrak{R}^{m}$ and $\boldsymbol{\lambda}$ belongs to $\mathfrak{R}^{p}$. Finally, let $\mathbf{J}(\mathbf{y} \mid \boldsymbol{\varepsilon})$ express the mapping on $\mathbf{K} \times \Re^{n+m+p}$ onto $\mathfrak{R}^{n+m+p}$ the components of which are as follows:

$$
\begin{gathered}
\mathbf{F}(\mathbf{x} \mid \mathcal{\varepsilon})-\sum_{\ell=1}^{m} \pi_{\ell} \nabla \mathrm{g}_{\ell}(\mathbf{x} \mid \mathcal{\varepsilon})-\sum_{\ell=1}^{p} \lambda_{\ell} \nabla \mathrm{d}_{\ell}(\mathbf{x} \mid \mathcal{\varepsilon}), \\
\pi_{\ell} \mathrm{g}_{\ell}(\mathbf{x} \mid \varepsilon) \text { for values of } \ell \text { between } 1 \text { and } m, \\
\mathrm{~d}_{\ell}(\mathbf{x} \mid \mathcal{E}) \text { for values of } \ell \text { between } 1 \text { and } p .
\end{gathered}
$$

Under some technical assumptions, at a solution point $\mathbf{x}^{*}$ in $\mathbf{K}$ there exist $\pi^{*}$ in $\Re^{m}$ and $\lambda^{*}$ in $\Re^{p}$ such that $\mathbf{J}\left(\mathbf{y}^{*} \mid \mathbf{0}\right)=\mathbf{0}$ and $\nabla_{\mathbf{y}} \mathbf{J}$ is invertible in a local neighbourhood of $\mathbf{y}^{*} \mid \mathbf{0}$, and that make the partial derivatives of $\mathbf{y}^{*}$ with respect to $\boldsymbol{\varepsilon}$ satisfy:

$$
\nabla_{\boldsymbol{\varepsilon}} \mathbf{y}^{*}=\left[\nabla_{\mathbf{y}} \mathbf{J}\left(\mathbf{y}^{*} \mid \mathbf{0}\right)\right]^{-1}\left[-\nabla_{\boldsymbol{\varepsilon}} \mathbf{J}\left(\mathbf{y}^{*} \mid \mathbf{0}\right)\right]
$$

The technical assumptions required to obtain (26) include the local uniqueness of a solution. However, in an assignment model it is seldom the case that the path flow equilibrium solution is unique. Tobin and Friesz (1988) also extended Tobin's results to address the case of non-uniqueness. The guidelines of their contribution are as follows:

i) assume the variational inequality problem involves a monotone mapping, and therefore has a convex solution set.

ii) select a nondegenerate, extreme point in this convex solution set and, at this point, develop the derivatives of the solution to a restricted problem for which uniqueness 
applies. In the restricted problem, only those paths which carry positive flow in the extreme point are considered.

iii) based on a strict complementary slackness condition, the solution to the disturbed, restricted problem is also a solution of the original disturbed problem (for small disturbances), and actually does not depend on the extreme point chosen.

\subsection{Propagation formulae for the dual criteria model}

In order to apply (26) to the dual criteria model, we must define the set $\mathbf{K}$, the mappings $\mathbf{F}, \mathbf{g}$ and $\mathbf{d}$ and the multipliers $\pi$ and $\lambda$. The notations $\mathbf{K}$ and $\mathbf{d}$ in sub-sections 2.3 and 2.4 are equivalent, therefore the vector $\mathbf{f}$ in sub-section 2.3 corresponds to the vector $\mathbf{x}$ in sub-section 2.4. The constraints $\mathbf{g}(\mathbf{x}) \geq \mathbf{0}$ in sub-section 2.4 correspond to both the non negativity conditions $\mathbf{f} \geq \mathbf{0}$ and the inequalities $\mathbf{z}(\mathbf{f}) \leq \mathbf{0}$, reformulated as $-\mathbf{z}(\mathbf{f}) \geq \mathbf{0}$. To make the mapping $\mathbf{U}$ of sub-section 2.3 correspond to the mapping $\mathbf{F}$ in sub-section 2.4, we have to meet Tobin and Friesz's requirements: to that end we shall from now assume that $\mathbf{U}$ is monotone. A sufficient condition for this is that the travel time mapping $\mathbf{f} \mapsto\left[\mathrm{T}_{i k}(\mathbf{f} ; \mathbf{0})\right]_{i k}$ be monotone, since the complementary terms $\mathrm{I}_{i k}-\mathrm{T}_{i k}$ derive from a convex function (Leurent, 1993a). Then the uniqueness of a solution to (23) holds with respect to each isoprice class flow. In the case of the dual criteria model, an extreme, nondegenerate solution point may have as many paths with positive flow as there are links and isoprice classes.

We shall break down the vector $\pi$ into two sub-vectors, $\mathbf{w}$ and $\overline{\mathbf{f}}$, with the respective components $w_{b}$ associated with the constraints $-\mathrm{z}_{b} \geq 0$ and $\bar{f}_{i k}$ which are linked to the non-negativity constraints $f_{i k} \geq 0$. Then, for a given disturbance $\varepsilon$, the components of the mapping $\mathbf{J}$ are as follows:

$$
\begin{gathered}
\mathbf{U}(\mathbf{f} \mid \varepsilon)-\overline{\mathbf{f}}+\sum_{b} w_{b} \nabla \mathrm{z}_{b}(\mathbf{f} \mid \varepsilon)-\sum_{\ell=1}^{\mathrm{N}_{d}} \lambda_{\ell} \nabla \mathrm{d}_{\ell}(\mathbf{f} \mid \varepsilon) \\
-w_{b} \cdot \mathrm{z}_{b}(\mathbf{f} \mid \mathcal{\varepsilon}) \text { for values of } b \text { between } 1 \text { and } \mathrm{N}_{\mathrm{b}} \\
\bar{f}_{i k} f_{i k} \text { for every path }(i) k \\
\mathrm{~d}_{\ell}(\mathbf{f} \mid \varepsilon) \text { for values of } \ell \text { between } 1 \text { and } \mathrm{N}_{\mathrm{d}} \text {. }
\end{gathered}
$$

To restrict the problem as in Tobin and Friesz (1988), we shall drop the path variables with zero flow at the equilibrium point. The condition set (27) is also reduced to (28):

$$
\begin{gathered}
\mathbf{U}(\mathbf{f} \mid \mathcal{E})+\sum_{b} w_{b} \nabla \mathrm{z}_{b}(\mathbf{f} \mid \mathcal{\varepsilon})-\sum_{\ell=1}^{\mathrm{N}_{d}} \lambda_{\ell} \nabla \mathrm{d}_{\ell}(\mathbf{f} \mid \mathcal{\varepsilon}), \\
-w_{b} \cdot \mathrm{z}_{b}(\mathbf{f} \mid \mathcal{E}) \text { for values of } b \text { between } 1 \text { and } \mathrm{N}_{b},
\end{gathered}
$$




$$
\mathrm{d}_{\ell}(\mathbf{f} \mid \mathcal{\varepsilon}) \text { for values of } \ell \text { between } 1 \text { and } \mathrm{N}_{d} \text {. }
$$

Let us now indicate closed-form expressions for the terms in $\nabla_{\mathbf{y}} \mathbf{J}$ and $\nabla_{\mathcal{E}} \mathbf{J}$. We denote the components of $\mathbf{J}$ in (28a) as $J_{i k}$, those in (28b) as $J_{b}$ and those in (28c) as $J_{\ell}$. Then: $\nabla_{\mathbf{y}} \mathbf{J}=$

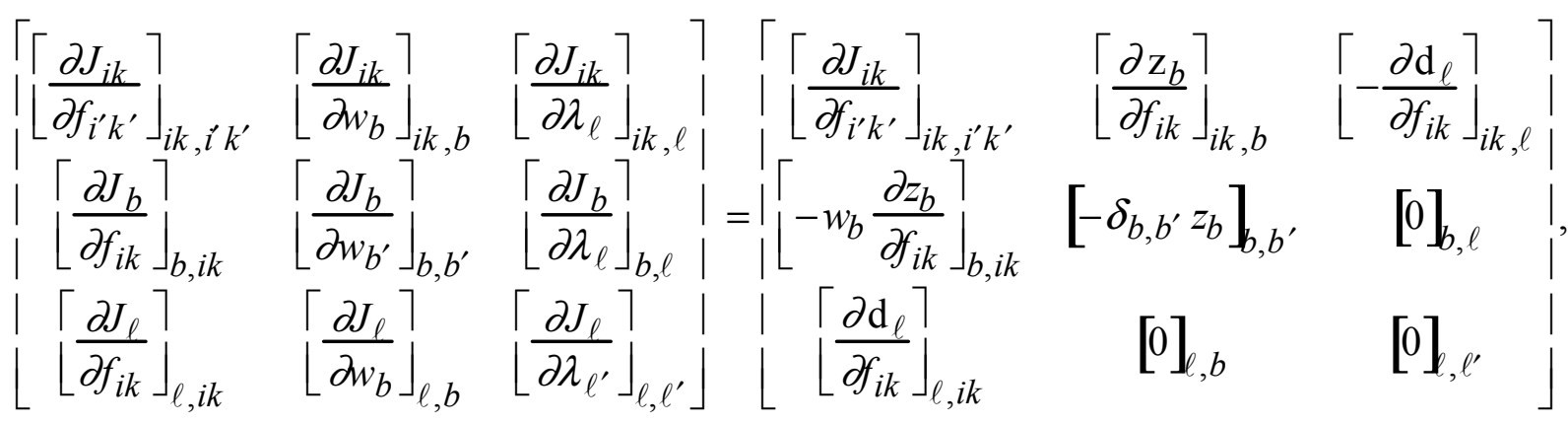

in which $\delta_{b, b^{\prime}}=1$ if $b=b^{\prime}$ or 0 otherwise.

From Leurent (1993a) we can state that:

$$
\begin{aligned}
\frac{\partial J_{i k}}{\partial f_{i^{\prime} k^{\prime}}}= & \left(\sum_{a} \delta_{i k}^{a} \frac{\partial \mathrm{t}_{a}}{\partial f_{i^{\prime} k^{\prime}}}\right)+\left(\sum_{b} w_{b} \frac{\partial^{2} \mathrm{z}_{b}}{\partial f_{i^{\prime} k^{\prime}} \partial f_{i k}}\right)-\left(\sum_{\ell} \lambda_{\ell} \frac{\partial^{2} \mathrm{~d}_{\ell}}{\partial f_{i^{\prime} k^{\prime}} \partial f_{i k}}\right) \\
& \left.\left.+\delta_{i, i^{\prime}} \mid-\frac{\partial \mathrm{D}_{i}^{-1}}{\partial q_{i}}+\sum_{n=1}^{m_{i}-1} \frac{\overline{\mathrm{P}}_{i n}-\overline{\mathrm{P}}_{i, n+1}}{q_{i}} \cdot\left(\Gamma_{i}^{n k}-\frac{q_{i n}^{\prime}}{q_{i}}\right) \cdot\left(\Gamma_{i}^{n k^{\prime}}-\frac{q_{i n}^{\prime}}{q_{i}}\right) \cdot \frac{\partial \eta_{i}^{\prime}(}{\partial \alpha} \frac{q_{i n}^{\prime}}{q_{i}}\right)\right),
\end{aligned}
$$

in which $\Gamma_{i}^{n k} \equiv \partial q_{i n}^{\prime} / \partial f_{i k}=1$ if the price of path $k$ is less than, or equal to, the price of the $n$-th isoprice class or $\Gamma_{i}^{n k}=0$ if it is not.

As regards $\nabla_{\varepsilon} \mathbf{J}$, for any given component $\varepsilon_{c}$ of $\boldsymbol{\varepsilon}$ we have that:

$$
\begin{aligned}
\frac{\partial J_{i k}}{\partial \varepsilon_{c}}= & \left(\sum_{a} \delta_{i k}^{a} \frac{\partial \mathrm{t}_{a}}{\partial \varepsilon_{c}}\right)+\left(\sum_{b} w_{b} \frac{\partial^{2} \mathrm{z}_{b}}{\partial \varepsilon_{c} \partial f_{i k}}\right)-\left(\sum_{\ell} \lambda_{\ell} \frac{\partial^{2} \mathrm{~d}_{\ell}}{\partial \varepsilon_{c} \partial f_{i k}}\right)-\frac{\partial \mathrm{D}_{i}^{-1}}{\partial \varepsilon_{c}}\left(q_{i}\right) \\
& +\overline{\mathrm{P}}_{i \bar{m}_{i}} \frac{\partial \eta_{i}}{\partial \varepsilon_{c}}(1)-\overline{\mathrm{P}}_{i 1} \frac{\partial \eta_{i}}{\partial \varepsilon_{c}}(0)+\sum_{n=1}^{\bar{m}_{i}-1}\left(\overline{\mathrm{P}}_{i n}-\overline{\mathrm{P}}_{i, n+1}\right)\left(\frac{\partial \eta_{i}}{\partial \varepsilon_{c}}\left(\frac{q_{i n}^{\prime}}{q_{i}}\right)-\frac{q_{i n}^{\prime}}{q_{i}} \cdot \frac{\partial \eta_{i}^{\prime}}{\partial \varepsilon_{c}}\left(\frac{q_{i n}^{\prime}}{q_{i}}\right)\right) \\
\frac{\partial J_{b}}{\partial \varepsilon_{c}}= & -w_{b} \frac{\partial \mathrm{z}_{b}}{\partial \varepsilon_{c}} \\
\frac{\partial J_{\ell}}{\partial \varepsilon_{c}}= & \frac{\partial \mathrm{d}_{\ell}}{\partial \varepsilon_{c}} .
\end{aligned}
$$

Thus the set of formulae required to conduct the sensitivity analysis of the dual criteria model is complete. To obtain the partial derivatives $\Xi$ of the outputs with respect to any disturbance $\varepsilon_{\mathcal{c}}$, one can solve the linear system $\left[\nabla_{\mathbf{y}} \mathbf{J}\right] \Xi=-\nabla_{\mathcal{\varepsilon}} \mathbf{J}$. 


\section{IMPLEMENTATION AND TEST}

In order to apply the method worked out in Section 2, a computer program was implemented and tested on a real-world example consisting of an interurban, motorway traffic study.

\subsection{A report of implementation}

A computer program has been implemented to address the case of a side constrained, elastic demand, dual criteria model with link travel time functions depending only on the flow on that link. The assignment algorithm consists of an outer loop to handle the side constraints by means of an augmented Lagrangian scheme (Larsson and Patriksson, 1994); inside the outer loop an unconstrained equilibrium assignment is performed, using the procedure of equalization by transfer which handles the paths. The procedure of equalization by transfer is based on the following principle: for a given O-D pair, the flow is transferred from the loaded path with the longest travel time to the shortest path in such a way as to equalize journey times or remove flow from the long path. This is performed first for one pair of paths and then for others, until the travel times on the paths reach equilibrium thereby ensuring that (identified) unused paths are not quicker. All O-D pairs are processed in this way and the algorithm iterates until convergence. To address the dual criteria model, the path travel times must be replaced by the path impedance functions (Leurent 1995a, b). A medium-size numerical experiment with the overall assignment procedure is reported in Leurent (1996a).

Based on the results of the assignment, viz. $\mathbf{f}^{*}$ and $\mathbf{w}^{*}$, the sensitivity and error analysis is performed in the following steps: (i) computation of $\nabla_{\mathbf{y}} \mathbf{J}$ and $\nabla_{\mathcal{E}} \mathbf{J}$, (ii) computation of $-\left[\nabla_{\mathbf{y}} \mathbf{J}\right]^{-1} \cdot \nabla_{\mathcal{E}} \mathbf{J}$ by means of a partial pivot-point method, (iii) for a given distribution of the exogenous error $\boldsymbol{\varepsilon}$, computation of the distribution of the propagated exogenous error on each positive path flow, (iv) on a pre-specified link $a_{0}$, aggregation of the error distribution of each path flow that contributes to the flow on that link. This yields the error distribution of the link flow $x_{a_{0}}$.

\subsection{Example data set}

We considered an interurban toll motorway which is part of one of the routes between Bordeaux and Pau in the south-west of France. These two cities are $220 \mathrm{~km}$ apart and are served by four main routes. For the purposes of the traffic study 180 one-way arcs and 19 demand zones were coded.

The assignment model was specified as follows. On the demand side, we considered two classes of vehicles, viz. passenger cars and heavy freight vehicles with an 
equivalency coefficient of 2 passenger car units. The VoT of the trips by car had a lognormal distribution, with respectively $\mu=2.48$ and $\sigma=0.6$ as the mean and standarddeviation of its natural logarithm. Hence the mean VoT of the trips by car was $\exp \left(\mu+\sigma^{2} / 2\right)=12 \$ / \mathrm{h}$. As regards the heavy freight vehicles, values of $\mu_{\mathrm{h}}=3.73$ and $\sigma_{\mathrm{h}}$ $=0.6$ were considered, ensuring a mean VoT of $50 \$ / \mathrm{h}$. For each traffic class and each O-D pair, the trip-rate was assumed to depend on the mean generalized travel time with constant elasticity -0.6 , i.e. $q_{i}=\mathrm{D}_{i}\left(\bar{S}_{i}\right)=q_{i}^{(0)}\left(\bar{S}_{i} / \bar{S}_{i}^{(0)}\right)^{-0.6}$.

On the supply side, no side constraints were considered. A BPR travel time function was assumed for each link, i.e. $\mathrm{t}_{a}\left(x_{a}\right)=t_{a}^{0} \cdot\left(1+0.15\left(\frac{x_{a}}{C_{a}}\right)^{4}\right)$ where $t_{a}^{0}$ is a free-flow travel time, $C_{a}$ is a practical capacity and $x_{a}$ is the link flow expressed in passenger car units. The travel cost on a link included the expense generated by fuel consumption and the toll if applicable, which is the case on most interurban motorways in France (with a toll rate of roughly $0.08 \$ / \mathrm{km}$ for passenger cars). The price of a given path was computed as the sum of the travel cost of the links that are incident to it. Note that this path travel time formula may be changed in our formulation of the dual criteria model, contrary to the formulation in Marcotte and Zhu (1994) and Dial (1996): this may be of use on modelling an O-D-cordon based toll scheme.

\subsection{Exogenous error and its propagation}

We selected a particular toll link, $a_{0}$, which is part of the A64 motorway near of Pau. We have studied the exogenous error $\varepsilon_{x_{a_{0}}}$ which is propagated for car traffic on $a_{0}$ by considering the a priori exogenous errors which relate to the following:

i) the journey times $\mathrm{t}_{a}$ on the arcs. The travel time of each arc $a$ has a centred gaussian error $\varepsilon_{T_{a}}$ with a standard deviation $\mathrm{s}_{T_{a}}$ such that $\mathrm{s}_{T_{a}} / T_{a}=10 \%$, and which is independent of other errors.

ii) the O-D volumes for car traffic. The reference demand volume $q_{i}^{(0)}$ of any O-D pair $i$ has a centred gaussian error $\varepsilon_{q_{i}^{(0)}}$ with a standard deviation $\mathrm{s}_{q_{i}^{(0)}}$ such that $\mathrm{s}_{q_{i}^{(0)}} / q_{i}^{(0)}=20 \%$, and which is independent from other errors.

iii) the parameters $\mu$ and $\sigma$ of a log-normal distribution of the value of time shared by all O-D pairs : $\mu$ is the mean and $\sigma$ the standard deviation of the logarithm of the value of time. $\mu$ and $\sigma$ are assumed to be subject to the centred gaussian uncertainties $\varepsilon_{\mu}$ and $\varepsilon_{\sigma}$, with standard deviations $\mathrm{s}_{\mu}$ and $\mathrm{s}_{\sigma}$ respectively such that $\mathrm{s}_{\mu} / \mu=1 \%$ and $\mathrm{s}_{\sigma} / \sigma=3 \% . \varepsilon_{\mu}$ and $\varepsilon_{\sigma}$ are assumed independent from other errors but correlated with each other according to $\operatorname{Cov}\left(\varepsilon_{\mu} ; \varepsilon_{\sigma}\right) / \mu \sigma=-0.1 \%$. 
On the studied road $a 0$, for an average modeled traffic $x_{a 0}$ of 1220 cars/day, after propagation of the a priori exogenous error we obtain an a posteriori error with mean zero and standard deviation $\mathrm{s}_{x_{a 0}}=123$ cars/day, hence $\mathrm{s}_{x_{a 0}} / x_{a 0}=10.1 \%$. This uncertainty is mainly due to demand volumes ( $83 \%$, see figure 4$)$ and to a lesser degree to journey times $(14 \%)$ and the distribution of values of time $(3 \%)$. The examples in Section 1 suggested that the absolute and relative sizes of the exogenous propagated errors are particular to each case. Here we may conclude that the overall propagated error is tolerably small, and that any attempt to reduce it should focus on the O-D flows. About the distribution of the VoT, the exogenous error on its parameters has a small effect because the whole distribution is considered and furthermore, as the parameters were estimated simultaneously (Leurent, 1996b), their respective exogenous errors are related and balance each other (through $\left.\operatorname{Cov}\left(\varepsilon_{\mu} ; \varepsilon_{\sigma}\right)\right)$.

\section{CONCLUSION}

The sensitivity analysis of the dual criteria model yields the derivatives of the equilibrium solution with respect to perturbation parameters. These parameters may be related to either the distribution of the value of time, the trip rates or the travel times. On the basis of the values of the derivatives and on probabilistic assumptions about the distribution of the exogenous error related to the parameters, the distribution of the exogenous error propagated on the model output can be assessed.

In this paper, an analytical method for sensitivity and error analysis was presented: this allows systematic, comprehensive "what if" analyses without having to solve one additional equilibrium problem for each disturbance parameter. The contribution of each parameter in the total output error may be evaluated: in cases where the revenue of a toll system is studied, it is of much use to the analyst to identify those parameters to which the revenue is most sensitive.

The method was demonstrated on a realistic example of an interurban motorway traffic study. Its results indicate that the exogenous error which relates to the trip rates contributes much more to the error on the toll revenue than that on the distribution of the value of time. This highlights the usefulness of the dual criteria model in which an error on the mean VoT is of less importance than in the single criterion model, since the whole distribution of the VoT is considered.

\section{ACKNOWLEDGMENTS}

The careful reading and wise advice of two anonymous referees are greatly appreciated. 


\section{REFERENCES}

Dafermos, S.C. (1981). A Multicriteria Route-Mode Choice Traffic Equilibrium Model. Unpublished manuscript, Lefschetz Center for Dynamical Systems, Brown University, Rhode Island.

Dial, R.B. (1996). Bicriterion Traffic Assignment: Basic Theory and Elementary Algorithms. Transportation Science, 30/2, 93-111.

Larsson, T., and Patriksson, M. (1994). An augmented Lagrangean Scheme for Capacitated Traffic Assignment Problems. In Actes INRETS Vol. 38, pp. 163-199. INRETS, Arcueil.

Leurent, F. (1993a). Cost versus Time Equilibrium over a Network. Eur. J. of Oper. Res., 71(2), 205-221.

Leurent, F. (1993b). Modelling Elastic, Disaggregate Demand. In Papageorgiou, M. et al, Proceedings of the First Meeting of the Euro Working Group on Urban Traffic and Transportation. Technical University of Munich, Munich.

Leurent, F.M. (1995a). The practice of a dual criteria assignment model with continuously distributed values-of-time. Proceedings of the 23rd European Transport Forum: Transportation Planning Methods, E, 117-128. PTRC, London.

Leurent, F.M. (1995b). Un algorithme pour résoudre plusieurs modèles d'affectation du trafic: la méthode d'égalisation par transvasement. Les Cahiers Scientifiques $d u$ Transport, 30, 31-49.

Leurent, F.M. (1996a). The Theory and Practice of a Dual Criteria Assignment Model with a Continuously Distributed Value-of-Time, in Transportation and Traffic Theory, Lesort J.B. (ed), 455-477. Pergamon, Exeter, England.

Leurent, F.M. (1996b). An analysis of modeling error, with application to a traffic assignment model with continuously distributed values of time. 24th European Transport Forum, Proceedings of Seminar D, 1. PTRC, London.

Leurent, F. (1997). Analyse et mesure de l'erreur dans un modèle de simulation. Thèse de Doctorat de l'ENPC. INRETS, Arcueil, France.

Marcotte, P., and Zhu, D. (1994). An efficient algorithm for a bicriterion traffic assignment problem. Preprints of the 3rd Capri Seminar, 2, 891-897. Capri, June.

Marche, R. (1973). Valuation of travel time, French inter-city values. Paper presented at the Symposium on Transportation and Environment, Southampton, April.

Quandt, R.E. (1968) Estimation of Modal Splits. Transportation Research, 2, 41-50. 
Smith, M.J. (1979). The Existence, Stability and Uniqueness of Traffic Equilibria. Transportation Research B, 13, 295-304.

Tobin, R.L. (1986). Sensitivity Analysis for Variational Inequalities. J. Optim. Theory Appl., 48, 191-204.

Tobin, R.L., and Friesz, T.L. (1988). Sensitivity Analysis for Equilibrium Network Flow. Transportation Science, 22/4, 242-250.

Tukey, J. W. (1957). The Propagation of Errors, Fluctuations and Tolerances: Basic Generalized Formulas. Dptt of Mathematics, Technical Report 10, Princeton University.

\section{APPENDIX: FORMULAE FOR A LOG-NORMAL VOT}

The dual criteria model can be applied with a log-normally distributed VoT $v$; if its natural logarithm has a mean $\mu$ and a standard deviation $\sigma$, then $\mathrm{H}(v)=\Phi\left(t_{v}\right)$ where $t_{v}=(\ln (v)-\mu) / \sigma$ and $\Phi$ is the CDF of a normal distribution with mean 0 and standard deviation 1 . Let $\phi$ be the probability density function of this normal distribution, i.e. $\phi(t)=\exp \left(-t^{2} / 2\right) / \sqrt{2 \pi}$. Then the VoT-related functions of interest in dual criteria assignment can be evaluated as:

$$
\begin{aligned}
& \begin{array}{l}
\lceil/ \partial v\rceil \\
\partial|/ \partial \mu| \mathrm{H}(v)=\frac{\phi\left(t_{v}\right)}{\sigma}\left|\begin{array}{c}
1 / v\rceil \\
-1 \\
/ \partial \sigma
\end{array}\right|,
\end{array} \\
& \mathrm{H}^{-1}(\alpha)=\exp \left(\mu+\sigma \Phi^{-1}(\alpha)\right) \text { and therefore } \eta^{\prime}(\alpha) \equiv 1 / \mathrm{H}^{-1}(\alpha)=\exp \left(-\mu-\sigma \Phi^{-1}(\alpha)\right) \\
& \eta(x)=\int_{0}^{x} \eta^{\prime}(\alpha) \mathrm{d} \alpha=\exp \left(\frac{\sigma^{2}}{2}-\mu\right) \Phi\left(\Phi^{-1}(x)-\sigma\right)
\end{aligned}
$$

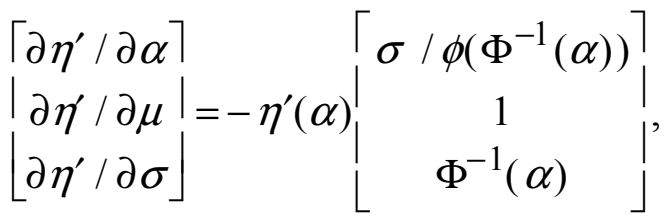

$$
\begin{aligned}
& \left.\mid \begin{array}{l}
\frac{\partial \eta}{\partial \mu}(x) \\
\mid \frac{\partial \eta}{\partial \sigma}(x)
\end{array}\right]=\left[\begin{array}{c}
-\eta(x) \\
\sigma \eta(x)-\exp \left(\frac{\sigma^{2}}{2}-\mu\right) \phi\left(\Phi^{-1}(x)-\sigma\right)
\end{array}\right] .
\end{aligned}
$$




\section{Figures}

Fig. 1. Model with fixed travel times and fixed demand

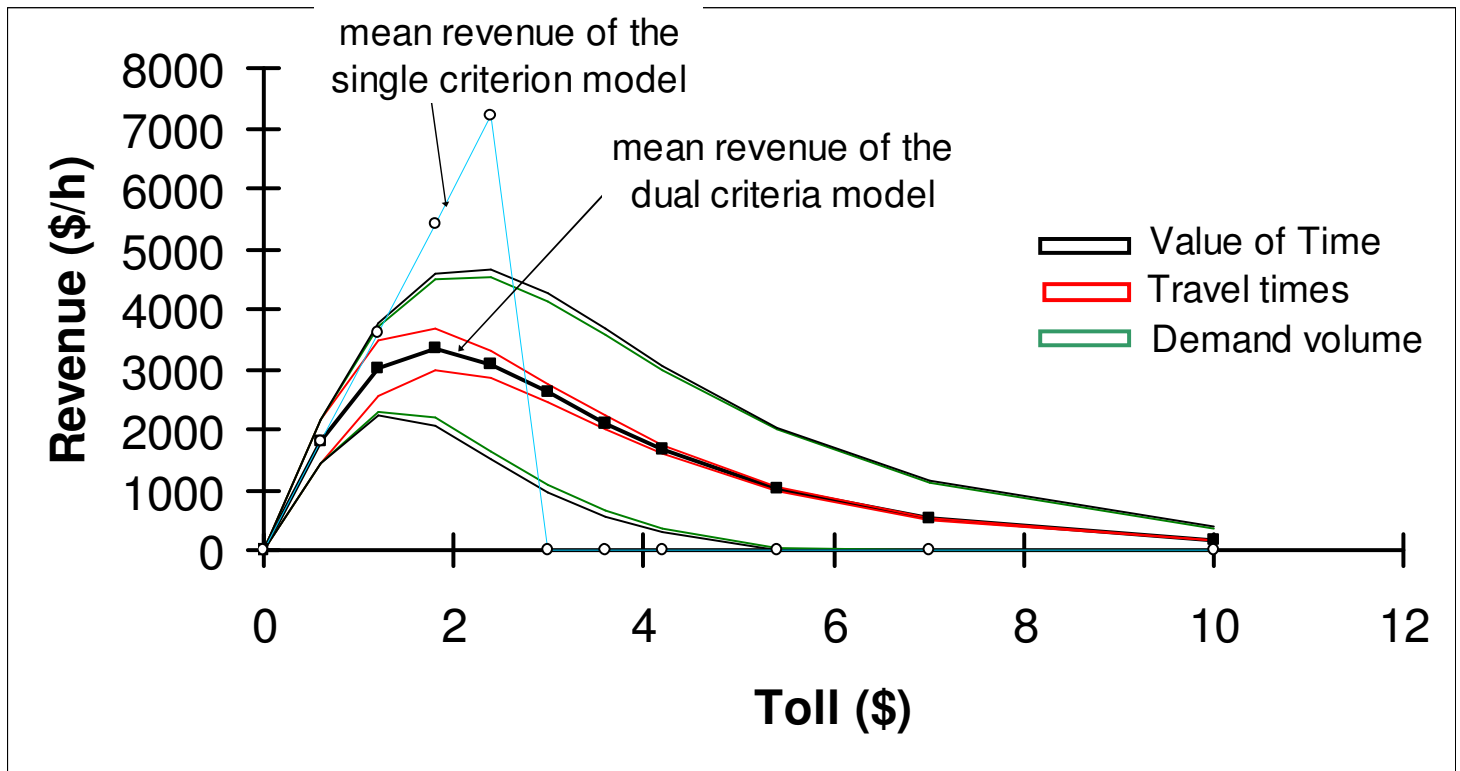

Fig. 2. Model with variable travel times and fixed demand

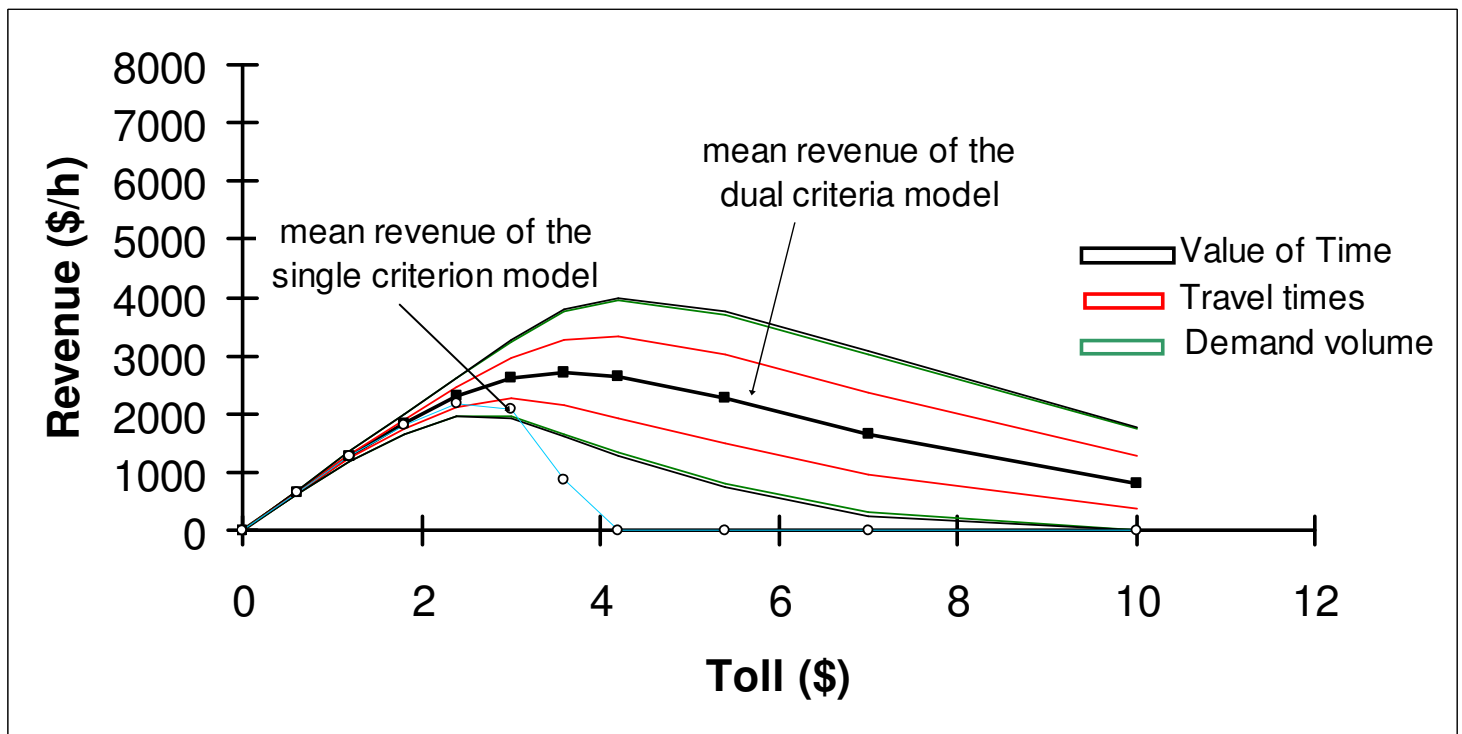


Fig. 3. Model with variable travel times and variable demand

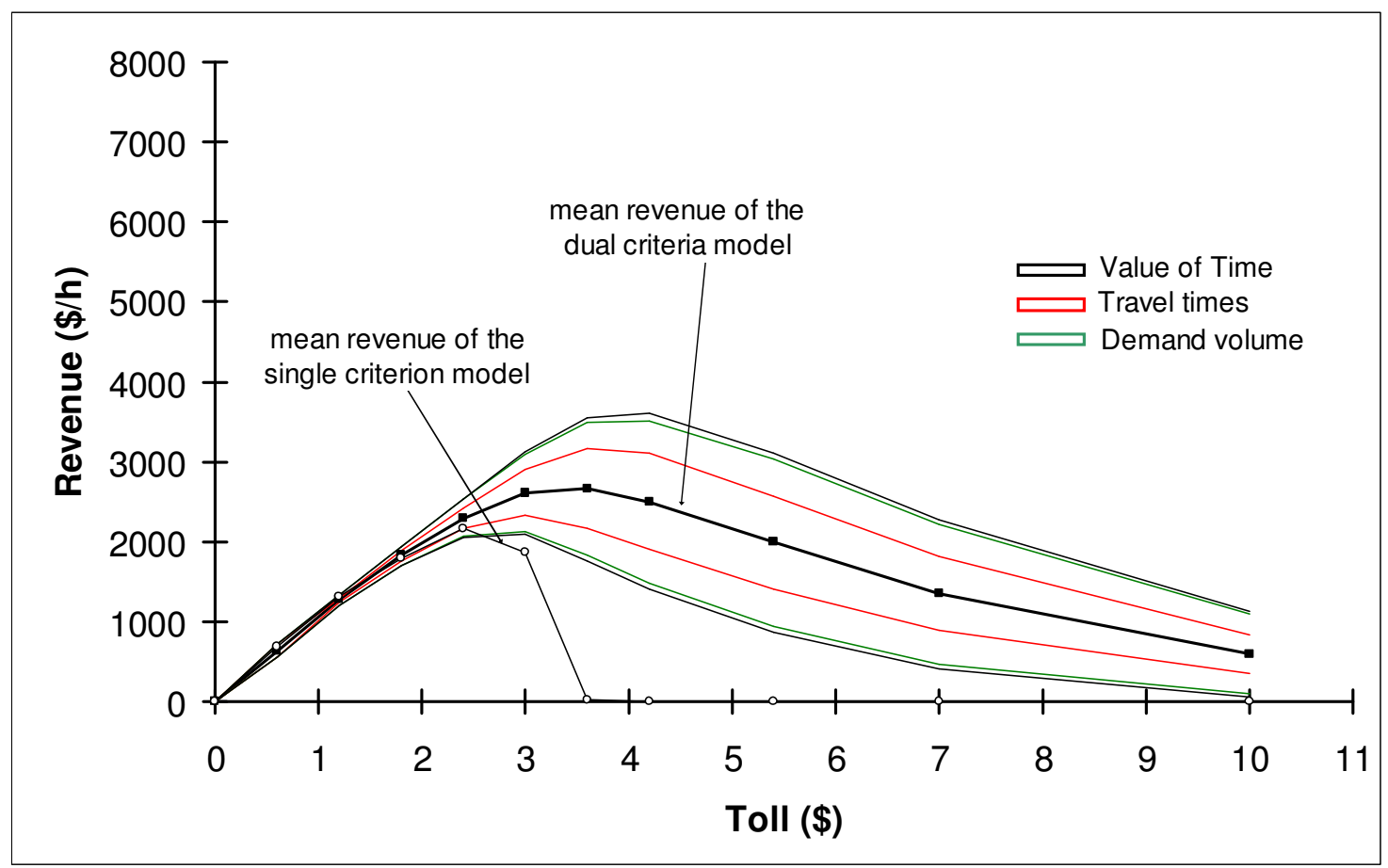

Fig. 4. The accumulation of exogenous uncertainties.

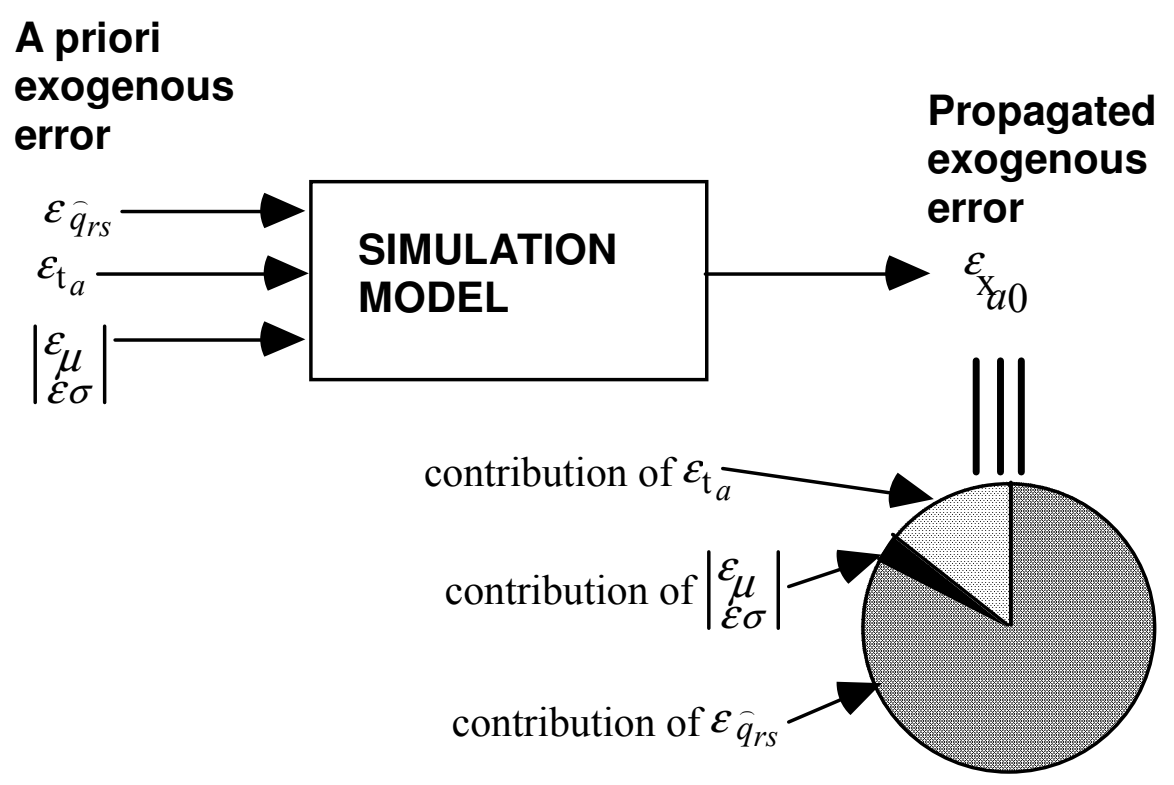

